\title{
A de novo matrix for macroscopic living materials from bacteria
}

Authors: Sara Molinari ${ }^{1}$, Robert F. Tesoriero Jr. ${ }^{1,2}$, Dong $\mathrm{Li}^{3}$, Swetha Sridhar ${ }^{1,2}$, Rong Cai ${ }^{1}$, Jayashree Soman ${ }^{1}$, Kathleen R. Ryan ${ }^{4}$, Paul D. Ashby ${ }^{3}$, Caroline M. Ajo-Franklinn,

\section{Affiliations:}

1. Department of Biosciences, Rice University, Houston, TX, USA.

2. Systems, Synthetic and Physical Biology PhD program, Rice University, Houston, TX, USA.

3. Molecular Foundry, Lawrence Berkeley National Laboratory, Berkeley, CA, USA

4. Plant \& Microbial Biology, University of California, Berkeley, Berkeley, CA, USA

* corresponding author. Email: cajo-franklin@rice.edu 


\section{Summary Paragraph:}

Engineered living materials (ELMs) are composites of living cells embedded in a biopolymer matrix that combine the desirable properties of natural biomaterials with non-natural, tailored properties. ELMs with a wide range of sophisticated biological functions have been created by engineering the embedded cells using synthetic biology. Engineering a de novo biomolecular matrix would offer control over material assembly, structure, and composition, thus enabling us to grow macroscopic ELMs with customizable mechanical properties. However, we have lacked the genetic tools and design rules to genetically encode a synthetic matrix that programs collective cell self-organization into macroscopic structures. Here we report growth of macroscopic ELMs from Caulobacter crescentus cells that display and secrete an engineered self-interacting protein. This protein formed an extracellular de novo matrix and assembled cells into hierarchicallyordered, centimeter-scale ELMs. We showed that the mechanical, catalytic, and morphological properties of these ELMs can be tuned through genetic modification of the matrix. Our work identifies novel genetic tools, design and assembly rules for growing macroscopic ELMs with both wide-ranging mechanical properties and customizable functions. We anticipate the modularity of this approach will permit the incorporation of different protein polymers in the de novo matrix, thus allowing to generate ELMs with a variety of desired structures and compositions of the bulk material. We envision specific matrix properties that can be combined synergistically with existing cellular functions to greatly expand the opportunities for ELMs in human health, energy, and the environment. 


\section{Main Text:}

Naturally occurring living biomaterials, such as bones or wood, grow bottom-up from a small number of progenitor cells into macroscale structures ${ }^{1}$. Engineered living materials $(\text { ELMs })^{2-4}$ are inspired by naturally-occurring living materials, but use synthetic biology to introduce tailored, non-natural functions. By incorporating engineered cells into a biopolymer matrix, these materials can function as living sensors ${ }^{5}$, therapeutics ${ }^{6}$, biomanfacturing platforms ${ }^{7}$, electronics $^{8}$, energy converters ${ }^{9}$, and structural materials ${ }^{10}$. While cells confer functionality to ELMs, the matrix assembles the material and controls the bulk material composition, structure, and function ${ }^{11}$.

Since the matrix plays such a key role over material properties, engineering macroscopic ELMs that grow from the bottom-up with a synthetic biomolecular matrix is a primary goal of the field. It is considered well beyond the current state-of-the art $^{11}$ because secreting recombinant biopolymers at concentrations that gelate is challenging ${ }^{12}$ and because assembly of micrometersized cells into centimeter-scale materials requires self-organization across length scales spanning four orders of magnitude. Engineering principles to achieve this are unknown ${ }^{11,12}$, so most ELMs are microscopic ${ }^{13-17}$ and must be processed into macroscopic materials. The few macroscopic ELMs have been created by genetically modifying existing matrices ${ }^{18}$ or genetically manipulating mineralization of inorganic matrices ${ }^{19}$. However, these approaches have afforded little genetic control over the matrix composition and only $\sim 20-30 \%$ changes in material mechanics ${ }^{18,19}$, which is much more limited than the tunability of naturally-occurring and chemically synthesized materials. 


\section{De novo engineering of a macroscopic bottom-up ELM}

Leveraging previous genetic tools for biopolymer secretion and display in Caulobacter crescentus $^{20,21}$, we sought to create bottom-up ELMs composed of cells interacting through a surface-bound de novo matrix. To minimize native cell-cell interactions, we started with a $C$. crescentus background that cannot form a biofilm. Next, we designed a bottom-upp de novo (BUD) protein by replacing the native copy of the surface layer (S-layer) RsaA ${ }^{22}$ (Fig. 1A) with a synthetic construct encoding four modules (Fig. 1B): (i) a surface-anchoring domain, (ii) a flexible biopolymer region for solution accessibility, (iii) a tag for functionalization, and (iv) a domain for secretion and self-interaction. We used the first 250 residues of RsaA as an anchor to the O-antigen lipopolysaccharide ${ }^{23,24}$. As the flexible domain, we chose an elastin-like polypeptide (ELP) based on human tropoelastin with 60 repeats of the Val-Pro-Gly-X-Gly motif ${ }^{21,25}$, ELP 60 . ELPs are flexible, self-associate, and form elastic structures. SpyTag ${ }^{26}$ was used as a functionalization tag, as it covalently binds to fusion proteins containing SpyCatcher. The C-terminal domain of the BUD protein, consisting of the last 336 residues of RsaA, was chosen to mediate protein secretion ${ }^{21}$ and to self-associate ${ }^{27}$. We refer to this BUD protein-expressing strain of $C$. crescentus as the BUD-ELM strain.

Surprisingly, cultures of the BUD-ELM strain yielded centimeter-scale, filamentous material (Fig. 1C - left) after $24 \mathrm{~h}$ of growth. The material contained intact C. crescentus cells (Fig. 1C-right), indicating these macroscopic materials were indeed BUD-ELMs. In contrast, the wild-type culture did not generate any visible aggregates (Fig. S1). To understand the role of BUD protein in this material, we compared the extracellular surface of planktonic cells of the BUDELM strain before material formation with other $C$. crescentus strains. When stained with SpyCatcher-GFP, cells of the BUD-ELM strain (Fig. 1D - left) showed GFP fluorescence (cyan) along the outer contour of the cells (yellow), demonstrating the BUD protein is on the extracellular 
surface. The BUD-ELM was not stained by free GFP (Fig. 1D - right), nor was the $\triangle$ SpyTag strain stained by SpyCatcher-GFP (Fig. S2A and B), confirming that staining required SpyTag and SpyCatcher. Atomic force microscopy (AFM) of the BUD-ELM cells showed a brush-like structure (Fig 1E - right) that distinguished it from the wild-type hexameric S-layer (Fig. 1E - left) and the $\triangle r s a A$ strain (Fig. 1E - middle). The BUD protein formed long, unstructured projections, and this soft layer mediates cell-cell interactions (Fig. S3). These results provide a first demonstration of macroscopic, bottom-up ELMs with a de novo surface-bound matrix that mediates cell-cell interactions.

A

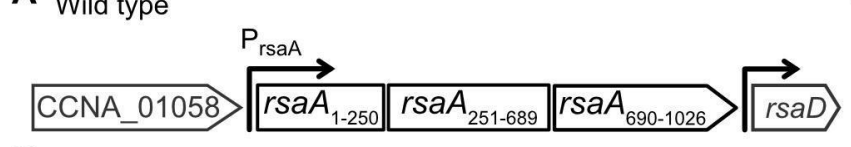

C

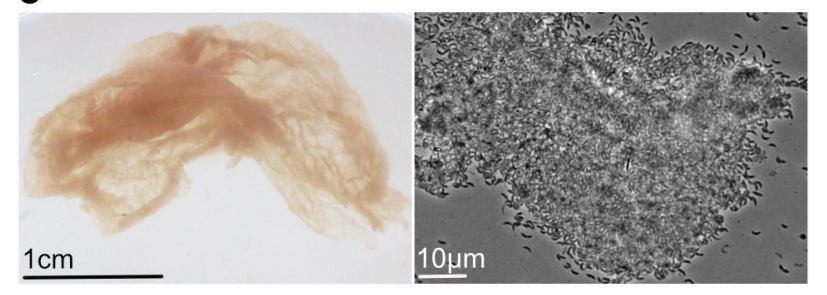

D

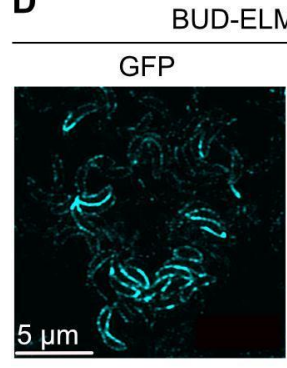

B BUD-ELMs strain

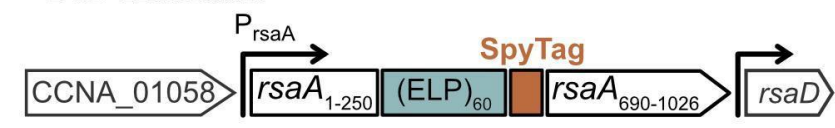

\section{$E$}
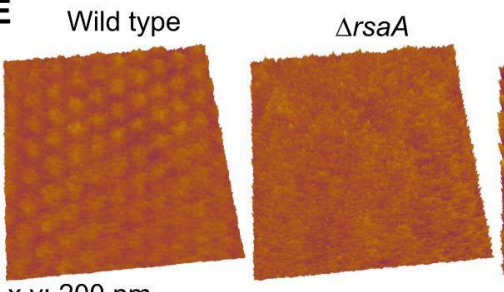

BUD-ELM strain

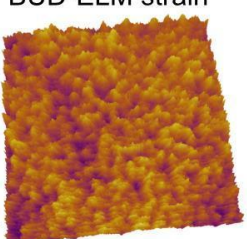

7.50

3.75

0

$-3.75$ $-7.50$ $x, y: 200 \mathrm{~nm}$ BUD-ELM strained with GFP

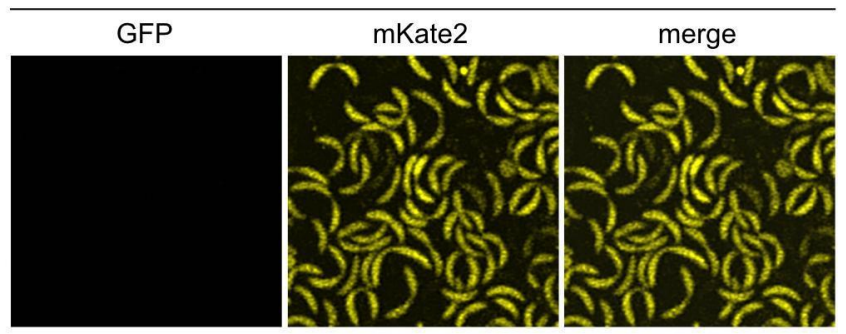

Fig. 1. Engineered strains of $\boldsymbol{C}$. crescentus self-assemble into BUD-ELMs. (A) Schematic of the native rsaA gene within its genomic context, showing its N-terminal cell anchoring domain (1-250) and C-terminal domain (251-1026) with the secretion subdomain (690-1026). (B) Schematic of the four-domain construct replacing the native $r s a A$ gene in the BUD-ELM strain. (C) Photograph of free-floating material formed by the BUD-ELM strain (left). Brightfield image of a portion of a BUD-ELM (right), showing cell clusters 
and intact cells. (D) Confocal microscopy of single cells of BUD-ELM strain stained with SpyCatcher-GFP (left) or GFP (right), demonstrating that the BUD protein (Fig. 2A - left) is located on the cell surface. Scale bar: $5 \mu \mathrm{m}$, applies to all images. (E) AFM images of the cell surface of wild-type (left), $\Delta r s a A$ (middle), and BUD-ELM strain (right), showing the brush-like structure of the BUD-ELM strain's surface.

\section{BUD-ELMs are organized hierarchically through a synthetic proteinaceous matrix}

To probe their structure, we stained BUD-ELMs with SpyCatcher-GFP and imaged them using confocal microscopy. At the half a millimeter length scale (Fig. 2A - left), the BUD protein (cyan) and cells (yellow) appear distributed throughout the entire material. At the micron length scale, C. crescentus cells in the material display a layer of BUD protein (Fig. 2A - right) like planktonic cells (Fig. 1D). However, at the tens of micron length scale (Fig. 2A - middle), we unexpectedly observed a BUD protein-containing secreted matrix (blue) that was locally inhomogeneous and was surrounded by C. crescentus cells (yellow) on all sides (Fig. S4). To probe the matrix composition, we also stained the BUD-ELM with Congo Red and 3,3'dioctadecyloxacarbocyanine perchlorate (DiO) (Fig. 2B), which are known to bind amyloid proteins $^{28}$ and lipids ${ }^{29}$, respectively. Congo Red staining (Fig. 2B - Proteins) was orthogonal to the cell channel and analogous to the SpyCatcher-GFP staining, confirming that the matrix is made of proteins. In contrast, DiO (Fig. 2B - Lipids), did not stain cell-free regions. Analysis of the cellfree and stained areas (Fig. 2C) confirmed that protein staining had a higher overlap with cellexcluded matrix regions compared to lipid staining. Thus, the BUD-ELM strain produces a secreted proteinaceous matrix containing the BUD protein that mediates BUD-ELM structure at the tens of micron length scale. 
To understand how the BUD protein could be both a surface-displayed and secreted matrix, we imaged single cells through AFM at early stages of BUD-ELM formation, when cells are mostly in the planktonic state, and at later stages when the material is fully assembled. At the early stage (Fig. 2E - left), the cell surface appeared uniform, but after the BUD-ELM had formed, cells showed large protuberances (Fig. 2E - right). Additionally, the surface layer depth of early-stage BUD-ELM cells is $\sim 10 \mathrm{~nm}$ (Fig. $2 \mathrm{~F}$ - left), compared to the $\sim 35 \mathrm{~nm}$ layer of late-stage cells (Fig. $2 \mathrm{~F}-$ right), indicating that the protein layer thickens over time. We found secreted BUD protein in the medium under both static and shaking conditions (Fig. 2D), indicating that some BUD protein is released into the medium independent of shaking. Moreover, a strain that only secretes, but does not display the BUD protein, created material with a much lower cell content than the BUD-ELM (Fig. S5). These results suggest that the BUD protein accumulates on the cell surface, some of which detaches and forms a secreted matrix that subsequently binds $C$. crescentus cells. We propose that the ability of cells to simultaneously self-interact and adhere to the matrix plays a pivotal role in creating an emergent structure that is cell-rich and macroscopic. 


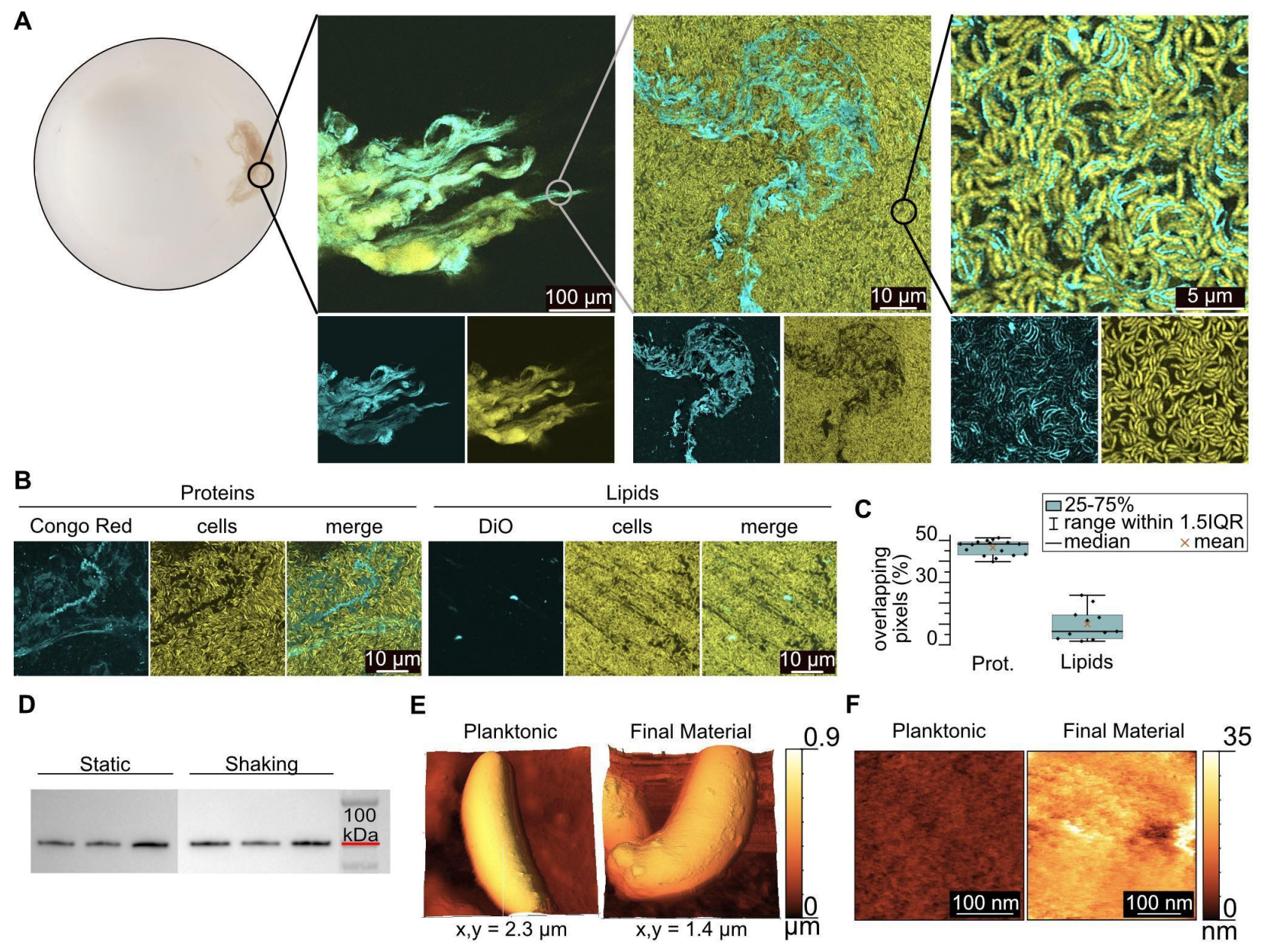

Fig. 2. BUD-ELMs contain a de novo protein matrix and display hierarchical structure. (A) Confocal microscopy of ELMs stained with SpyCatcher-GFP at increasing magnifications, showing a hierarchical structure. Bottom images show individual fluorescent channels: GFP (matrix) on the left and mKate2 (cells) on the right. (B) Confocal microscopy of BUD-ELMs stained with Congo Red (left panel) and DiO (right panel), highlighting the proteinaceous nature of the BUD protein matrix. (C) Percentage of overlapping pixels between cell-free and stained regions, confirming the absence of lipids in the BUD protein matrix. Error bars represent standard error of 16 images for the protein and 11 for the lipid staining. (D) Immunoblot of BUD protein in growth media in static (left) and shaking (right) cultures. (E) AFM images of single cells at early (left) and late (right) stages of BUD-ELM formation, showing a difference in surface morphology. (F) High-resolution AFM images of single-cell surfaces at early (left) and late (right) stages of BUD-ELM formation, showing differences in surface layer thickness. 
We next sought to understand how this material assembles by imaging BUD-ELM cultures at various times during their growth (Fig. 3A). Shaken cultures grew planktonically for $\sim 12 \mathrm{~h}$ (Fig. 3A - left) before a thin pellicle appeared at the air-water interface (Fig. 3A - middle). AFM images of the pellicle depicted a central, cell-dense region (Fig. 3B - left) and a peripheral region of a few cells attached to a $\sim 6 \mathrm{~nm}$ thick membrane (Fig. 3B - middle and right), suggesting the BUD protein forms a protein membrane to which cells adhere. The pellicle increased in density and opacity, becoming more compact. After $\sim 24 \mathrm{~h}$ total culturing time, the pellicle desorbed from the air-water interface and sank as the final material (Fig. 3A - right). Disrupting the hydrophobicity of the air/water interface by the addition of surfactant prevented pellicle and material formation (Fig. S6). Similarly, neither a pellicle nor material formed under static growth conditions. However, when static cultures were shaken, a pellicle formed (Fig. S7). Together, these experiments demonstrate that BUD-ELMs are formed through a multi-step process and establish hydrophobicity of the air/water interface and shaking as critical conditions for assembly of BUDELMs.

\section{BUD-ELMs are formed through a multi-step process that depends on physical parameters}

To understand how physical parameters affect BUD-ELM assembly, we grew cultures under different conditions and measured the size of the resulting materials. The size of BUD-ELMs depended non-monotonically on the shaking speed, volume, and flask diameter (Fig. 3C). We then used these parameters to calculate two quantities: the volumetric power input, $\mathrm{P}_{\mathrm{v}}$, describing the energy provided to the flask by shaking per unit volume and the volumetric mass transfer coefficient, $\mathrm{k}_{\mathrm{La}}$, representing the transfer of oxygen into the medium relative to the area of the airwater interface. We found that neither parameter showed a consistent relationship with the size of 
BUD-ELMs per flask (Fig. S8). Instead, we found empirically that the product of $\mathrm{P}_{\mathrm{V}}, \mathrm{k}_{\mathrm{La}}$, and the fifth power of the flask diameter, which we refer to as the modified volumetric power parameter, $\mathrm{P}_{\mathrm{V}, \mathrm{A}}$, related the culture conditions to the material size (Fig. 3D). With these data, we propose a model for BUD-ELM assembly (Fig. 3E). During culturing, the BUD protein accumulates in solution and on the surface of $C$. crescentus. With shaking, the BUD protein adsorbs to the airwater interface to form a protein-rich membrane of increasing thickness. BUD protein-displaying cells adhere to the membrane, increasing its density to form a pellicle. Hydrodynamic forces from shaking cause the pellicle to collapse on itself, until the material sinks to the bottom of the flask. At lower $\mathrm{P}_{\mathrm{V}, \mathrm{A}}$ values, the weaker hydrodynamic forces do not collapse the pellicle, and the pellicle fragments into smaller materials. At intermediate $\mathrm{P}_{\mathrm{V}, \mathrm{A}}$ values, stronger hydrodynamic forces collapse the pellicle into a single, large BUD-ELM. At higher $\mathrm{P}_{\mathrm{V}, \mathrm{A}}$ values, shear forces prevent the assembly of larger pellicles, leading to smaller pellicles and final materials. This empirical model provides a basis for the future development of mechanistic models describing BUD-ELM assembly. 


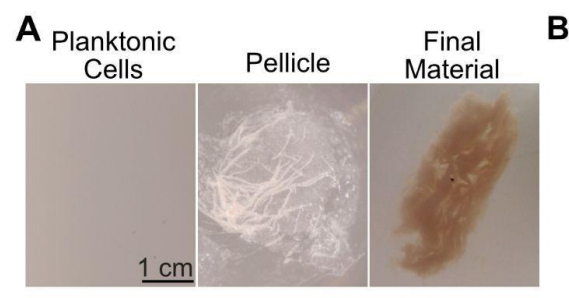

C

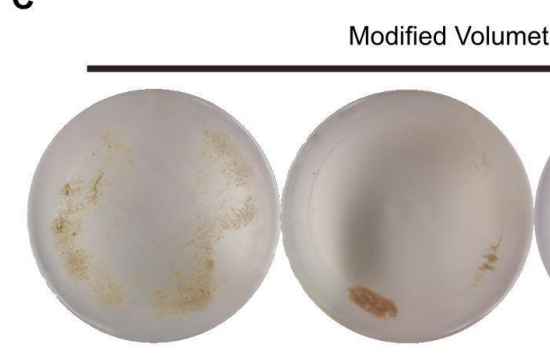

E

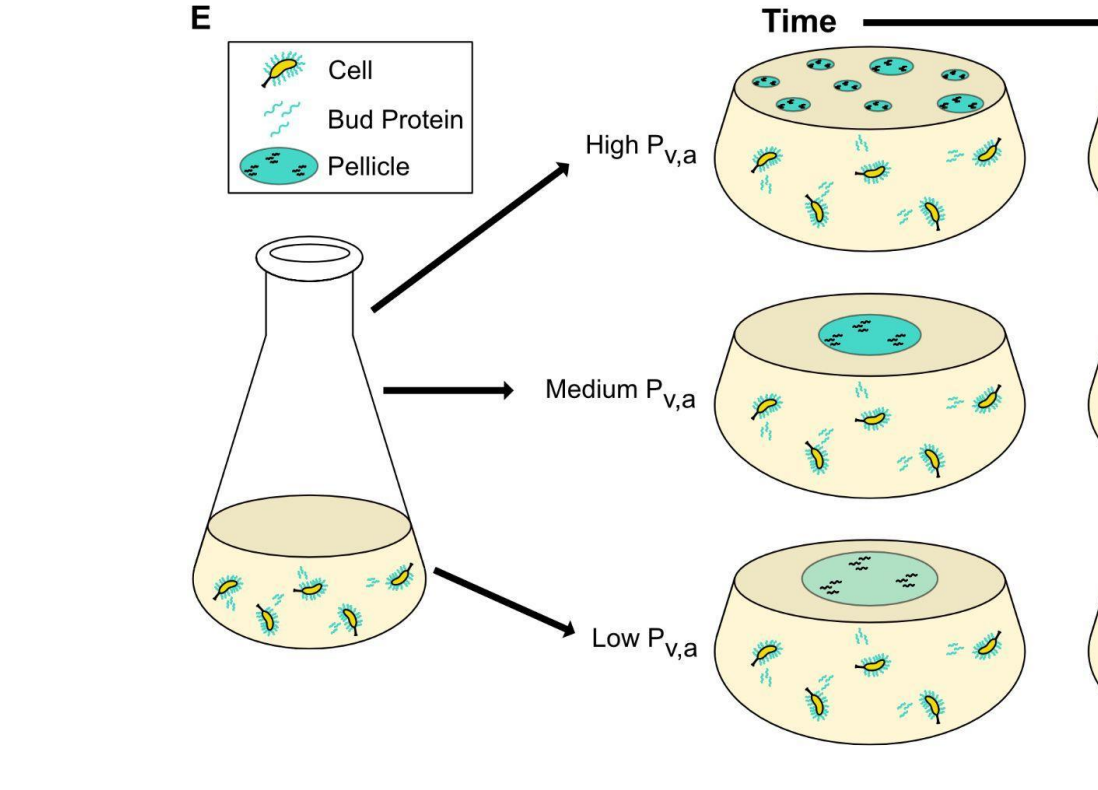

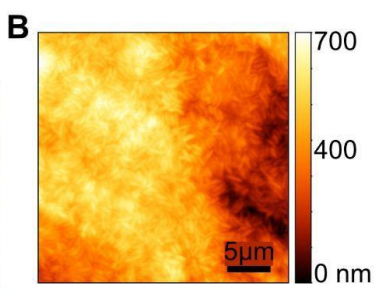
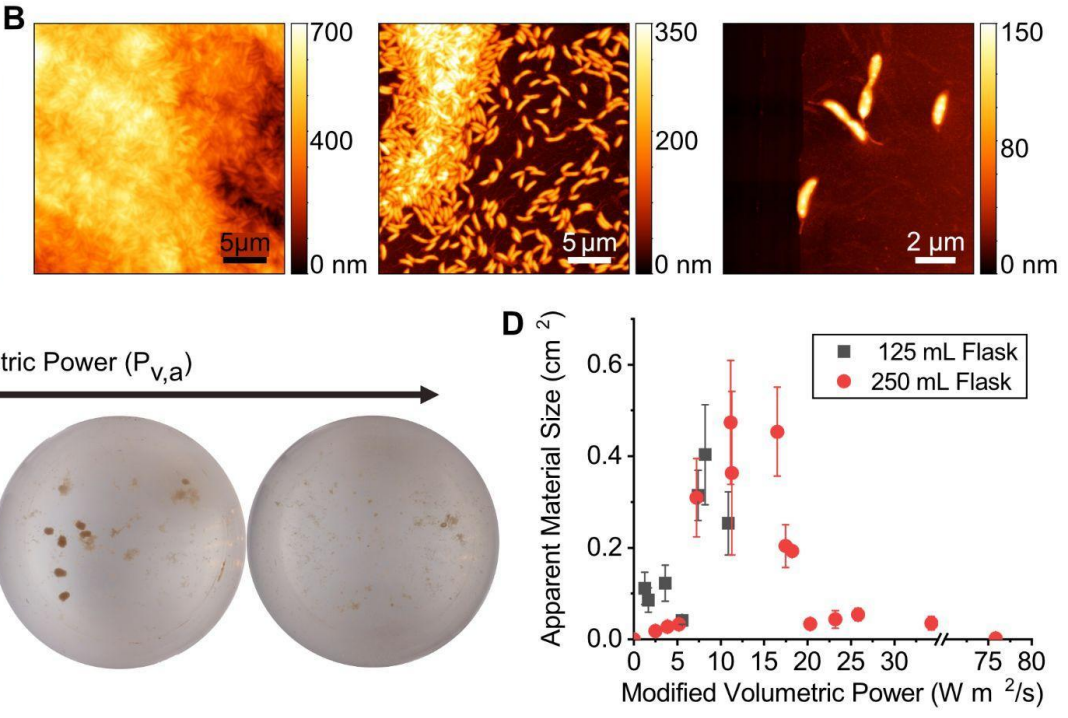
Modified Volumetric Power $\left(\mathrm{W} \mathrm{m}^{2} / \mathrm{s}\right)$
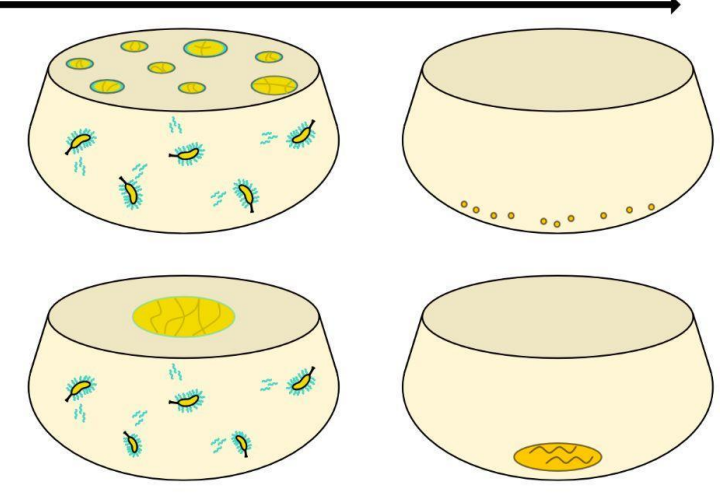

Fig. 3. BUD-ELMs are formed through a shaking-dependent, multi-step process. (A) Optical images of representative BUD-ELM strain culture during material, showing BUD-ELMs are formed through a multi-step process. (B) AFM images of pellicle structure, showing the pellicle contains both a central region containing several layers of densely packed cells (left), and a peripheral region containing sparse cells connected by a thin membrane (center and right). (C) Representative optical images of BUD-ELMs grown under different modified volumetric power values. Altering the modified volumetric power changes the morphology and size of the BUD-ELMs. (D) Correlation between modified volumetric power and the apparent surface area of the BUD-ELMs for 125- and 250-mL shake flasks. Error bars represent standard error of at least three samples. (E) Proposed mechanism for BUD-ELM formation. 


\section{BUD-ELMs are self-regenerating, multifunctional materials whose mechanical properties can be tuned genetically}

Since the matrix plays an important role in determining the mechanics of biomaterials, and the matrix of BUD-ELMs is mostly constituted by the BUD proteins, we hypothesized that genetic manipulations of the BUD protein will have a significant effect on the mechanical properties of BUD-ELMs. To test this, we interrogated the effects of removing the flexible biopolymer domain (strain $\triangle E L P_{60} \Delta S p y T a g$, Fig. $4 \mathrm{~A}$ - top) and the anchoring domain (strain $\Delta r s a A_{1-250}$, Fig. S5A) from the original BUD-ELM strain. We observed that both the $\triangle E L P_{60} \Delta S p y T a g$ (Fig. S5B - right) and $\Delta r s a A_{1-250}$ strain (Fig. 4A - bottom) form BUD-ELMs, that are morphologically distinguishable from the original BUD-ELM material in the flask after growth. However, despite these apparent differences, rheological measurements (Fig. S9) confirm that all three BUD-ELMs are viscoelastic solids. Frequency sweep curves (Fig. S10) show large and significant differences in the storage modulus $\left(\mathrm{G}^{\prime}\right)$ and the loss modulus $\left(\mathrm{G}^{\prime \prime}\right)$ among the three BUD-ELMs throughout the tested range of angular frequency. For a central value of angular frequency of $10 \mathrm{rad} / \mathrm{s}$ (Fig. 4B), the storage modulus of $\triangle E L P_{60} \Delta$ SpyTag BUD_ELMs is $\sim 340 \%$ of the original BUD-ELM, whereas the loss modulus increased by $\sim 300 \%$. Conversely, the $\Delta r s a A_{1-250}$ BUD-ELMs show a lower G' and G', respectively $69 \%$ and $84 \%$ less than the original BUD-ELM. We speculate that the increased stiffness of the $\triangle E L P_{60} \Delta S p y T a g$ BUD-ELMs reflects the removal of a long flexible linker, the ELP, from the cohesive BUD protein forming this cellular material. On the other end, we suggest $\Delta r s a A_{1-250}$ BUD-ELMs are less stiff due to the lack of crosslinking among cells and between the matrix and the cells. Overall, these results demonstrate that we can control BUDELMs mechanical properties over a 16-fold range through genetic modification of the matrixforming BUD-protein. 
ELMs must be able to be processed and stored without losing their ability to regrow. We dried BUD-ELMs (Fig. 4C - left and middle) and re-inoculated fragments of them into fresh medium (Fig. 4C - right). BUD-ELM fragments dried for 7, 14, or 21 days regenerated to form additional BUD-ELMs (Fig. 4D). Whereas BUD-ELMs re-grew 100\% of the time after 7 or 14 days of drying, BUD-ELMs desiccated for 21 days regenerated in 33\% of cases. Additionally, BUD-ELMs collected from multiple cultures formed a cohesive paste (Fig. 4E - top) that was extrudable through syringes with different diameters (Fig. 4E - bottom, left and middle). When mixed with glass powder, BUD-ELMs created a firmer paste that hardened into a solid composite (Fig. 4E - bottom, right). These results indicate that BUD-ELMs can regenerate after drying, can be reshaped, and can be processed into composite materials.

Lastly, we probed the ability of BUD-ELMs to behave as functional materials. Selfregenerating materials that remove heavy metals from water could help address the growing prevalence of heavy metal contamination. Since many forms of biomass non-specifically absorb heavy metals, we hypothesized that the BUD-ELM could remove $\mathrm{Cd}^{2+}$ from solution. When $0.013 \pm 0.007 \mathrm{~g}$ of $\triangle S p y T a g$ BUD-ELM was incubated for 90 min with a $\mathrm{CdCl}_{2}$ solution of $6 \mathrm{ppb}-$ $1 \mathrm{ppb}$ above the Environmental Protection Agency (EPA) limit-90 $\pm 5 \%$ of cadmium was removed (Fig. 4F). Next, we functionalized the BUD-ELM matrix to allow it to perform biological catalysis. We fused the oxidoreductase PQQ-glucose dehydrogenase (GDH), which couples oxidation of glucose to reduction of a soluble electron carrier $^{30}$, to SpyCatcher. Cell lysates containing overexpressed apo SpyCatcher-GDH or GDH were reconstituted by adding the cofactor PQQ (pyrroloquinoline quinone) to obtain the holo forms of the enzyme. After confirming the activity of holo GDH in both cases, we observed that only BUD-ELMs incubated with SpyCatcher-holoGDH enzymatically reduced an electron carrier (Fig. 4G and Fig. S12A). This demonstrates that 
BUD-ELM can be functionalized directly from complex mixtures to act as catalysts. Together, these results show BUD-ELMs can serve as versatile functional materials.

A
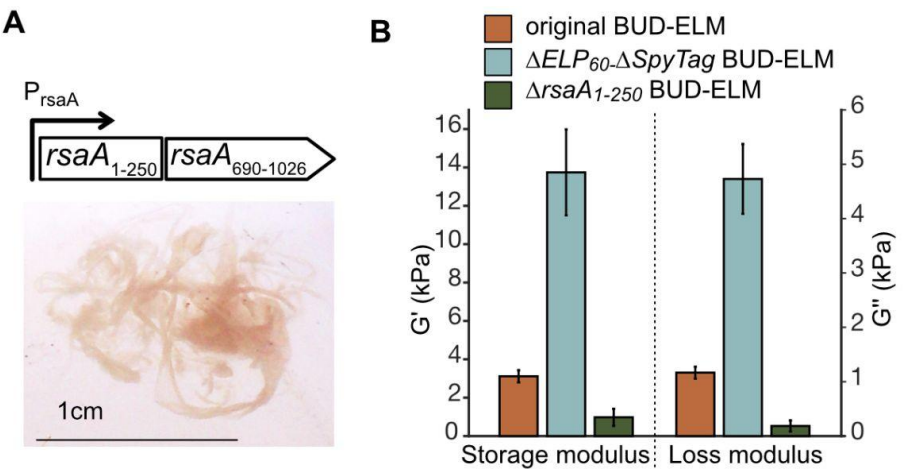

E

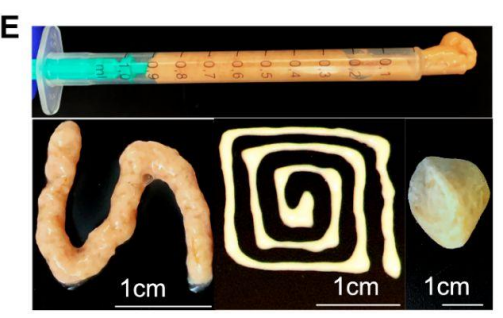

F

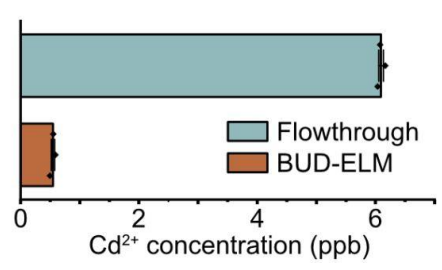

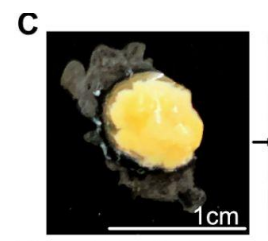

D
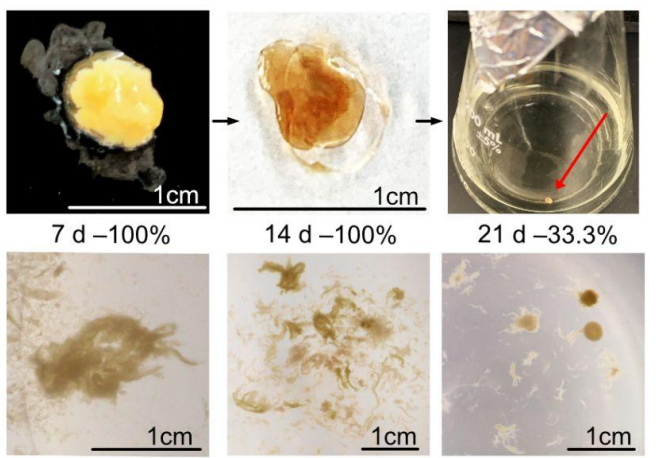

G

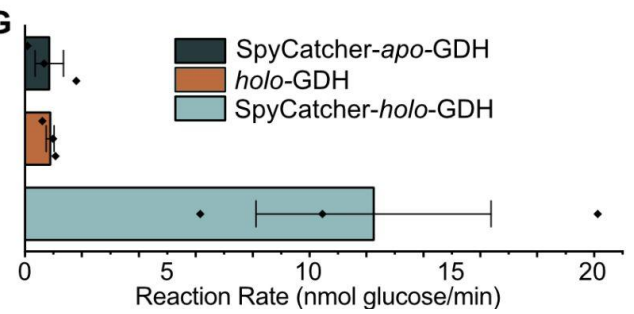

Fig. 4. BUD-ELMs are self-regenerating, processible, and functional materials. (A) Genetic constructs replacing the native $r s a A$ gene in the $\triangle E L P_{60} \Delta \operatorname{SpyTag}$ (left), and image of corresponding BUD-ELM (right). (B) Storage $\left(\mathrm{G}^{\prime}\right)$ and Loss $\left(\mathrm{G}^{\prime \prime}\right)$ modulus of original, $\Delta E L P_{60} \Delta$ SpyTag and $\Delta r s a A_{1-250}$ BUD-ELMs at an angular frequency of $10 \mathrm{rad} \mathrm{s}^{-1}$. Error bars represent 95\% confidence intervals of at least five samples. (C) Representative example of BUD-ELMs reseeding, showing extraction from liquid culture (left), desiccated (middle) and inoculation into fresh medium (right). (D) Representative example of BUD-ELMs grown from desiccated material after 7 (left), 14 (middle) or 21 (right) days. The percentage of successful BUD-ELM regeneration was $100 \%, 100 \%$ and $33.3 \%$ respectively. Percentages are calculated from at least nine samples. (E) BUD-ELMs collected into a syringe (top) for extrusion using different-sized nozzles, (bottomleft and bottom-middle), showing their ability to be reshaped. BUD-ELMs mixed with glass powder to form a firm paste that hardens when dehydrated (bottom-right), showing its potential as cement-like agent. (F) Graph showing the final $\mathrm{Cd}^{2+}$ solution concentration after 6 ppb $\mathrm{Cd}^{2+}$ solution was incubated with (BUDELM) or without (flowthrough) the $\triangle$ SpyTag BUD-ELMs. Error bars represent standard error of three 
samples. (G) Graph showing the rate of glucose oxidation for BUD-ELMs that were incubated with SpyCatcher-holo-GDH, holo-GDH, or SpyCatcher-apo-GDH. Error bars represent standard error of three samples.

In this work, the serendipitous creation of macroscopic ELMs allowed us to identify a new design principle - that macroscale BUD-ELMs are associated with a secreted extracellular matrix. We suggest that cell-matrix interactions may be essential for BUD-ELMs to reach a macroscopic size. This idea is supported by previous literature that shows that neither cell-cell adhesion alone ${ }^{31}$, nor sparse cell-matrix interactions in the absence of additional forces ${ }^{19}$ lead to microscopic cell aggregates. Additionally, we have demonstrated that nucleation of a pellicle at the liquid-air interface and hydrodynamically-driven coalescence and collapse of the pellicle are required to form macroscopic ELMs. Since pellicle formation is also a key step in nanocellulose-based living materials $^{18}$, we suggest that the use of the air-water interface to locally concentrate and order hydrophobic biomolecules into a matrix may represent a general assembly principle for macroscopic ELMs. The new tools and C. crescentus platform developed here will permit systematic exploration of design and assembly rules for programming the growth of centimeterscale structures using living cells as building blocks.

By creating BUD-ELMs with a de novo, modular protein matrix, this work greatly expands the ability to tailor macroscopic ELMs for specific applications. Existing examples of macroscopic, bottom-up ELMs have extracellular matrices predominantly composed of polysaccharides, allowing little control over composition or mechanics ${ }^{18}$. Here we show genetic changes to a single engineered protein can yield dramatic changes in the ELM's composition and mechanical properties. The modularity of the BUD protein and the ease of engineering protein biopolymers offer much greater opportunities for introducing desirable properties into the matrix ${ }^{11}$. 
Known polypeptides and proteins can exhibit desirable optical, electrical, mechanical, thermal, transport, and catalytic properties ${ }^{32}$. We envision specific matrix properties that can be combined synergistically with existing cellular functions such as sensing, biomolecule production, and information processing. Thus, this work multiplies the opportunities to program ELMs tailored for applications in human health, energy, and the environment.

\section{References:}

1. Wegst, U. G. K., Bai, H., Saiz, E., Tomsia, A. P. \& Ritchie, R. O. Bioinspired structural materials. Nature Mater 14, 23-36 (2015).

2. Nguyen, P. Q., Courchesne, N. M. D., Duraj-Thatte, A., Praveschotinunt, P. \& Joshi, N. S. Engineered Living Materials: Prospects and Challenges for Using Biological Systems to Direct the Assembly of Smart Materials. Advanced Materials 30, 1704847-1704847 (2018).

3. Srubar, W. V. Engineered Living Materials: Taxonomies and Emerging Trends. Trends in Biotechnology 39, 574-583 (2021).

4. Tang, T.-C. et al. Materials design by synthetic biology. Nature Reviews Materials 1-19 (2020) doi:10.1038/s41578-020-00265-w.

5. Liu, X. et al. 3D Printing of Living Responsive Materials and Devices. Advanced Materials 30, 1704821 (2018).

6. González, L. M., Mukhitov, N. \& Voigt, C. A. Resilient living materials built by printing bacterial spores. Nature Chemical Biology 16, 126-133 (2020).

7. Dai, Z. et al. Versatile biomanufacturing through stimulus-responsive cell-material feedback. Nat Chem Biol 15, 1017-1024 (2019).

8. Krawczyk, K. et al. Electrogenetic cellular insulin release for real-time glycemic control in type 1 diabetic mice. Science 368, 993-1001 (2020). 
9. Yong, Y.-C., Yu, Y.-Y., Zhang, X. \& Song, H. Highly Active Bidirectional Electron Transfer by a Self-Assembled Electroactive Reduced-Graphene-Oxide-Hybridized Biofilm. Angewandte Chemie International Edition 53, 4480-4483 (2014).

10. Pungrasmi, W., Intarasoontron, J., Jongvivatsakul, P. \& Likitlersuang, S. Evaluation of Microencapsulation Techniques for MICP Bacterial Spores Applied in Self-Healing Concrete. Sci Rep 9, 12484 (2019).

11. Molinari, S., Tesoriero, R. F. \& Ajo-Franklin, C. M. Bottom-up approaches to engineered living materials: Challenges and future directions. Matter 4, 3095-3120 (2021).

12. Gilbert, C. \& Ellis, T. Biological Engineered Living Materials: Growing Functional Materials with Genetically Programmable Properties. ACS Synth. Biol. 8, 1-15 (2019).

13. Wang, H. et al. Genetical Surface Display of Silicatein on Yarrowia lipolytica Confers Living and Renewable Biosilica-Yeast Hybrid Materials. ACS Omega 5, 7555-7566 (2020).

14. Dorval Courchesne, N.-M., Duraj-Thatte, A., Tay, P. K. R., Nguyen, P. Q. \& Joshi, N. S. Scalable Production of Genetically Engineered Nanofibrous Macroscopic Materials via Filtration. ACS Biomater. Sci. Eng. 3, 733-741 (2017).

15. Chen, A. Y. et al. Synthesis and patterning of tunable multiscale materials with engineered cells. Nature Mater 13, 515-523 (2014).

16. Chen, B. et al. Programmable living assembly of materials by bacterial adhesion. Nat Chem Biol 1-6 (2021) doi:10.1038/s41589-021-00934-z.

17. Wang, Y. et al. Living materials fabricated via gradient mineralization of light-inducible biofilms. Nat Chem Biol 17, 351-359 (2021).

18. Gilbert, C. et al. Living materials with programmable functionalities grown from engineered microbial co-cultures. Nature Materials 1-10 (2021) doi:10.1038/s41563-020-00857-5. 
19. Kang, S.-Y. et al. Engineering Bacillus subtilis for the formation of a durable living biocomposite material. Nat Commun 12, 7133 (2021).

20. Charrier, M. et al. Engineering the S-Layer of Caulobacter crescentus as a Foundation for Stable, High-Density, 2D Living Materials. ACS Synthetic Biology 8, 181-190 (2019).

21. Orozco-Hidalgo, M. T. et al. Engineering High-Yield Biopolymer Secretion Creates an Extracellular Protein Matrix for Living Materials. mSystems 6, e00903-20.

22. Bharat, T. A. M. et al. Structure of the hexagonal surface layer on Caulobacter crescentus cells. Nature Microbiology 2, 1-6 (2017).

23. Walker, S. G., Karunaratne, D. N., Ravenscroft, N. \& Smit, J. Characterization of mutants of Caulobacter crescentus defective in surface attachment of the paracrystalline surface layer. Journal of Bacteriology 176, 6312-6323 (1994).

24. von Kügelgen, A. et al. In Situ Structure of an Intact Lipopolysaccharide-Bound Bacterial Surface Layer. Cell 180, 348-358.e15 (2020).

25. Roberts, S., Dzuricky, M. \& Chilkoti, A. Elastin-like polypeptides as models of intrinsically disordered proteins. FEBS Letters 589, 2477-2486 (2015).

26. Zakeri, B. et al. Peptide tag forming a rapid covalent bond to a protein, through engineering a bacterial adhesin. PNAS 109, E690-E697 (2012).

27. Bingle, W. H., Nomellini, J. F. \& Smit, J. Secretion of the Caulobacter crescentusS-Layer Protein: Further Localization of the C-Terminal Secretion Signal and Its Use for Secretion of Recombinant Proteins. Journal of Bacteriology 182, 3298-3301 (2000).

28. Yakupova, E. I., Bobyleva, L. G., Vikhlyantsev, I. M. \& Bobylev, A. G. Congo Red and amyloids: history and relationship. Bioscience Reports 39, BSR20181415 (2019).

29. Majewska, A., Yiu, G. \& Yuste, R. A custom-made two-photon microscope and deconvolution system. Pflügers Arch - Eur J Physiol 441, 398-408 (2000). 
30. Lisdat, F. PQQ-GDH - Structure, function and application in bioelectrochemistry.

Bioelectrochemistry 134, 107496 (2020).

31. Glass, D. S. \& Riedel-Kruse, I. H. A Synthetic Bacterial Cell-Cell Adhesion Toolbox for Programming Multicellular Morphologies and Patterns. Cell 174, 649-658.e16 (2018).

32. Kaplan, D. \& McGrath, K. Protein-Based Materials. (Springer Science \& Business Media, 1997).

Acknowledgements: We thank Marimikel Charrier, Maria Orozco Hidalgo, and Dr. Vera Troselj for helpful conversations.

Funding: This work was primarily supported by the Defense Advanced Research Projects Agency (Engineered Living Materials Program, C.M.A-F.). Additional support was provided by Cancer Prevention and Research Institute of Texas (RR190063, C.M.A-F.) and Office of Naval Research (N00014-21-1-2362, C.M.A-F.). Work at the Molecular Foundry was supported by the Office of Science, Office of Basic Energy Sciences, of the U.S. Department of Energy under Contract No. DE-AC02-05CH11231.

\section{Author contributions:}

Conceptualization: SM, RFT, DL, CAF, KRR, PDA

Methodology: SM, RFT, RC, SS, JS, DL, CAF

Investigation: SM, RFT, RC, SS, JS, DL

Visualization: SM, RFT, RC, DL

Funding acquisition: CAF, KRR, PDA

Project administration: CAF 
Supervision: SM, CAF

Writing - original draft: SM, CAF, RFT

Writing - review \& editing: SM, CAF, RFT, RC, SS, JS, DL, KRR, PDA

Competing interests: The authors declare no competing interests.

Data and materials availability: All strains and plasmids can be supplied upon reasonable request.

\section{Supplementary Materials}

Materials and Methods

Figs. S1 to S12

Tables S1 to S3 


\section{A de novo matrix for macroscopic living materials from bacteria}

Sara Molinari ${ }^{1}$, Robert F. Tesoriero ${ }^{1,2}$, Dong Li $^{3}$, Swetha Sridhar ${ }^{1,2}$, Rong Cai ${ }^{1}$, Jayashree Soman ${ }^{1}$,

Kathleen R. Ryan ${ }^{4}$, Paul D. Ashby ${ }^{3}$, Caroline M. Ajo-Franklin ${ }^{1, *}$.

Correspondence to: cajo-franklin@rice.edu

\section{This document includes:}

Materials and Methods

Figs. S1 to S12

Tables S1 to S3 


\section{Materials and Methods}

\section{Construction of C. crescentus strains}

All C. crescentus strains used in this work are listed in Table S1.

To create the $C$. crescentus strains, we cloned integration plasmids designed to incorporate synthetic genes into the rsaA locus using homologous recombination. For the BUD-ELM strain (RCC002), we created integration plasmid pSMCAF008 by inserting a target sequence into the multicloning site of the backbone plasmid pNPTS138 (GenBank: MK533795.1) using restriction enzymes (ApaI upstream and NheI downstream). The target sequence (ordered from GenScript, sequence below) contained the DNA sequence encoding the ELP $60-S p y T a g$ flanked by 800 bp of homology regions up- and downstream of the native $r s a A$ central domain ( $r s a A 750-2073)$. These flanking regions allow the synthetic gene to be properly positioned within the $C$. crescentus genome. The $\Delta E L P_{60} \Delta$ SpyTag and $\triangle$ SpyTag strains (RCC004 and RCC005) were generated with integration plasmids pSMCAF017 and pSMCAF018. These plasmids were cloned by PCR amplification of pSMCAF008 using the primers listed below and Golden Gate assembly.

The wild type strain of C. crescentus (MFm126) was transformed with pSMCAF008, pSMCAF017 and pSMCAF018, to generate RCC002, RCC003, and RCC004 strains, respectively, using a two-step recombination technique ${ }^{20}$. The two-step recombination technique with sucrose counterselection is as follows: the pNPTS138 plasmids were electroporated into E. coli WM3064 cells and subsequently conjugated overnight into C. crescentus NA1000 AsapA::Pxyl-mkate2 (MFm126) on a PYE agar plate containing 300n $\mu$ M DAP. The culture was then plated on PYE with kanamycin to select for integration of the plasmid and removal of E. coli cells. Successful integrants were incubated in liquid PYE media overnight and plated on PYE supplemented with 
$3 \% \mathrm{w} / \mathrm{v}$ sucrose to select for excision of the plasmid and $s a c B$ gene, leaving the target sequence in the genome. Integration of the sequences was confirmed by colony PCR (with primers SMCAF078 and SMCAF079) using a Touchdown thermocycling protocol with an annealing temperature ranging from $72-62^{\circ} \mathrm{C}$, decreasing $1^{\circ} \mathrm{C}$ per cycle. The PCR amplicons have been later sequenced (with primers SMCAF093 and SMCAF095);

Primers used to verify the synthetic strains:

SMCAF078: CTTAGTCTAGCGATCCTCGCCTAG

SMCAF079: ATCGCTGCTCCCATGCGC

SMCAF093: GTCCTTGTAGTCACCCGAG

SMCAF095: AGTTGCAGAGCCGTGAAG

Primers used to assemble pSMCAF017:

SMCAF142: TGGGTCTCAAGGGCGGTTCGGGAGGAGGC

SMCAF143: TGGGTCTCAGCAATCCAAACGAGAGTCTAATAGAATGAGGTC

SMCAF144: TGGGTCTCCTTGCAACTGGTCTATTTTCCTCTTTTG

SMCAF145: TAGGTCTCCCCCTTGTCATCGTCGTCCTTG

Primers used to assemble pSMCAF018:

SMCAF168: CAGGTCTCTTGTCGCACCTGATTGCCCGA

SMCAF169: CAGGTCTCTGCTGGGACACCACCGCCAGG

SMCAF170: CTGGTCTCCCAGCTGACCCGGCCTTCGGC

SMCAF171: CTGGTCTCTGACAATCTATCGATTGTATGGGAAGCCCG 
GenScript target sequence:

CCAATGATCGTAATACGACTCACTAGTGGGGCCCGCGCCACTCGGTCGCAGGGGGT GTGGGATTTTTTTTGGGAGACAATCCTCATGGCCTATACGACGGCCCAGTTGGTGAC TGCGTACACCAACGCCAACCTCGGCAAGGCGCCTGACGCCGCCACCACGCTGACGC TCGACGCGTACGCGACTCAAACCCAGACGGGCGGCCTCTCGGACGCCGCTGCGCTG ACCAACACCCTGAAGCTGGTCAACAGCACGACGGCTGTTGCCATCCAGACCTACCA GTTCTTCACCGGCGTTGCCCCGTCGGCCGCTGGTCTGGACTTCCTGGTCGACTCGAC CACCAACACCAACGACCTGAACGACGCGTACTACTCGAAGTTCGCTCAGGAAAACC GCTTCATCAACTTCTCGATCAACCTGGCCACGGGCGCCGGCGCCGGCGCGACGGCTT TCGCCGCCGCCTACACGGGCGTTTCGTACGCCCAGACGGTCGCCACCGCCTATGACA AGATCATCGGCAACGCCGTCGCGACCGCCGCTGGCGTCGACGTCGCGGCCGCCGTG GCTTTCCTGAGCCGCCAGGCCAACATCGACTACCTGACCGCCTTCGTGCGCGCCAAC ACGCCGTTCACGGCCGCTGCCGACATCGATCTGGCCGTCAAGGCCGCCCTGATCGGC ACCATCCTGAACGCCGCCACGGTGTCGGGCATCGGTGGTTACGCGACCGCCACGGC CGCGATGATCAACGACCTGTCGGACGGCGCCCTGTCGACCGACAACGCGGCTGGCG TGAACCTGTTCACCGCCTATCCGTCGTCGGGCGTGTCGGGTTCGGGCGGTTCGGGAG GAGGCTCGGGTGACTACAAGGACGACGATGACAAGGGAGTTGGCGTCCCAGGAGTT GGAGTCCCAGGAGGGGGCGTTCCGGGCGCAGGAGTTCCTGGAGTAGGAGTTCCAGG AGTGGGCGTGCCAGGGGTGGGCGTCCCAGGTGGGGGAGTTCCCGGAGCAGGTGTGC CTGGGGGCGGCGTGCCTGGAGTCGGAGTTCCGGGGGTGGGTGTACCGGGTGGAGGC GTACCAGGCGCGGGAGTGCCGGGCGTGGGCGTGCCAGGCGTCGGTGTACCCGGCGT TGGTGTTCCGGGCGGAGGTGTCCCCGGAGCTGGGGTTCCCGGTGGGGGTGTACCGG GCGTCGGGGTTCCCGGTGTGGGTGTCCCAGGTGGCGGCGTTCCCGGGGCGGGCGTA 
CCTGGAGTGGGTGTGCCAGGAGTCGGCGTCCCAGGAGTCGGCGTACCAGGAGGTGG TGTTCCCGGGGCCGGAGTTCCCGGCGGAGGAGTTCCCGGCGTCGGCGTCCCTGGGGT CGGCGTCCCGGGAGGTGGAGTACCCGGAGCAGGAGTGCCGGGAGTCGGTGTACCTG GTGTCGGTGTCCCTGGTGTAGGTGTCCCGGGTGGTGGGGTGCCAGGTGCTGGCGTAC CTGGGGGGGGGGTTCCTGGCGTAGGCGTTCCGGGGGTGGGCGTTCCGGGCGGCGGG GTGCCGGGAGCAGGTGTCCCCGGCGTTGGTGTACCGGGGGTTGGTGTCCCAGGCGT AGGTGTGCCCGGTGGAGGGGTGCCGGGAGCTGGAGTGCCTGGAGGGGGTGTACCAG GGGTCGGTGTTCCCGGTGTAGGAGTACCGGGGGGCGGAGTCCCAGGAGCCGGCGTG CCGGGTGTTGGAGTCCCGGGAGTCGGAGTCCCTGGGGTAGGCGTTCCAGGGGGAGG GGTCCCCGGTGCAGGGGTTCCTGGCGGTGGTGTCCCAGGCGGTTCGGGAGGAGGCT CGGGTGCGCATATCGTAATGGTCGATGCATACAAGCCCACGAAAGGAGGTTCAGGC GGCGGAAGCGGTGGTGGAAGCGGAGGTGGGTCAGGCGGAGGCTCAGGGGGAGGTT CGGGTGGCGGTTCGGGAGGAGGCTCGGGTGCTGACCCGGCCTTCGGCGGCTTCGAA ACCCTCCGCGTCGCTGGCGCGGCGGCTCAAGGCTCGCACAACGCCAACGGCTTCAC GGCTCTGCAACTGGGCGCGACGGCGGGTGCGACGACCTTCACCAACGTTGCGGTGA ATGTCGGCCTGACCGTTCTGGCGGCTCCGACCGGTACGACGACCGTGACCCTGGCCA ACGCCACGGGCACCTCGGACGTGTTCAACCTGACCCTGTCGTCCTCGGCCGCTCTGG CCGCTGGTACGGTTGCGCTGGCTGGCGTCGAGACGGTGAACATCGCCGCCACCGAC ACCAACACGACCGCTCACGTCGACACGCTGACGCTGCAAGCCACCTCGGCCAAGTC GATCGTGGTGACGGGCAACGCCGGTCTGAACCTGACCAACACCGGCAACACGGCTG TCACCAGCTTCGACGCCAGCGCCGTCACCGGCACGGGCTCGGCTGTGACCTTCGTGT CGGCCAACACCACGGTGGGTGAAGTCGTCACGATCCGCGGCGGCGCTGGCGCCGAC TCGCTGACCGGTTCGGCCACCGCCAATGACACCATCATCGGTGGCGCTGGCGCTGAC 
ACCCTGGTCTACACCGGCGGTACGGACACCTTCACGGGTGGCACGGGCGCGGATAT CTTCGATATCAACGCTATCGGCACCTCGACCGCTTTCGTGACGATCACCGACGCCGC TGTCGGCGACAAGCTCGACCTCGTCGGCATCTCGACGAACGGCGCTATCGCTGACG GCGCCTTCGGCGCTGCGGTCACCCTGGGCGCTGCTGCGACGCTAGCTGACTGGGAA AACCCTGGCGTTAATCGGAAAGAACATGTGAGCAAAAGGCCAGCAAAAGGCCAGG AACCGTAAAAAGGCCGCGTTGCTGGCGTTTTTCCATAGGCTCCGCCCCCCTGACGAG CATCACAAAAATCGACGCTCAAGTCAGAGGTGGCGAAACCCGACAGGACTATAAAG ATACCAGGCGTTTCCCCCTGGAAGCTCCCTCGTGCGCTCTCCTGTTCCGACCCTGCCG CTTACCGGATACCTGTCCGCCTTTCTCCCTTCGGGAAGCGTGGCGCTTTCTCATAGCT CACGCTGTAGGTATCTCAGTTCGGTGTAGGTCGTTCGCTCCAAGCTGGGCTGTGTGC ACGAACCCCCCGTTCAGCCCGACCGCTGCGCCTTATCCGGTAACTATCGTCTTGAGT CCAACCCGGTAAGACACGACTTATCGCCACTGGCAGCAGCCACTGGTAACAGGATT AGCAGAGCGAGGTATGTAGGCGGTGCTACAGAGTTCTTGAAGTGGTGGCCTAACTA CGGCTACACTAGAAGAACAGTATTTGGTATCTGCGCTCTGCTGAAGCCAGTTACCTT CGGAAAAAGAGTTGGTAGCTCTTGATCCGGCAAACAAACCACCGCTGGTAGCGGTG GTTTTTTTGTTTGCAAGCAGCAGATTACGCGCAGAAAAAAAGGATCTCAAGAAGATC CTTTGATCTTTTCTACGGGGTCTGACGCTCAGTGGAACGAAAACTCACGTTAAGGGA TTTTGGTCATGAGATTATCAAAAAGGATCTTCACCTAGATCCTTTTAAATTAAAAAT GAAGTTTTAAATCAATCTAAAGTATATATGAGTAAACTTGGTCTGACAGTTACCAAT GCTTAATCAGTGAGGCACCTATCTCAGCGATCTGTCTATTTCGTTCATCCATAGTTGC CTGACTCCCCGTCGTGTAGATAACTACGATACGGGAGGGCTTACCATCTGGCCCCAG TGCTGCAATGATACCGCGAGACCCACGCTCACCGGCTCCAGATTTATCAGCAATAAA CCAGCCAGCCGGAAGGGCCGAGCGCAGAAGTGGTCCTGCAACTTTATCCGCCTCCA 
TCCAGTCTATTAATTGTTGCCGGGAAGCTAGAGTAAGTAGTTCGCCAGTTAATAGTT TGCGCAACGTTGTTGCCATTGCTACAGGCATCGTGGTGTCACGCTCGTCGTTTGGTAT GGCTTCATTCAGCTCCGGTTCCCAACGATCAAGGCGAGTTACATGATCCCCCATGTT GTGCAAAAAAGCGGTTAGCTCCTTCGGTCCTCCGATCGTTGTCAGAAGTAAGTTGGC CGCAGTGTTATCACTCATGGTTATGGCAGCACTGCATAATTCTCTTACTGTCATGCCA TCCGTAAGATGCTTTTCTGTGACTGGTGAGTACTCAACCAAGTCATTCTGAGAATAG TGTATGCGGCGACCGAGTTGCTCTTGCCCGGCGTCAATACGGGATAATACCGCGCCA CATAGCAGAACTTTAAAAGTGCTCATCATTGGAAAACGTTCTTCGGGGCGAAAACTC TCAAGGATCTTACCGCTGTTGAGATCCAGTTCGATGTAACCCACTCGTGCACCCAAC TGATCTTCAGCATCTTTTACTTTCACCAGCGTTTCTGGGTGAGCAAAAACAGGAAGG CAAAATGCCGCAAAAAAGGGAATAAGGGCGACACGGAAATGTTGAATACTCATACT CTTCCTTTTTCAATATTATTGAAGCATTTATCAGGGTTATTGTCTCATGAGCGGATAC ATATTTGAATGTATTTAGAAAAATAAACAAATAGGGGTTCCGCGCACATTTCCCCGA AAAGTGCCACCTGACGTC

\section{Construction of Escherichia coli strains for protein overexpression}

To create E. coli strains that overexpressed GFP, GFP-Spycatcher, GDH, and GDHSpyCatcher, we first constructed plasmids coding for these proteins under control of an arabinose inducible promoter. Plasmids pSMCAF015 and pSMCAF016 were assembled from previously constructed $\mathrm{pBAD}-\mathrm{RFP}^{20}$ and $\mathrm{pBAD}-\mathrm{SpyCatcher-RFP}{ }^{20}$ plasmids respectively, by substituting the mRFP sequence with the GFP sequence. Similarly, plasmids pSMCAF032 and pSMCAF029 were

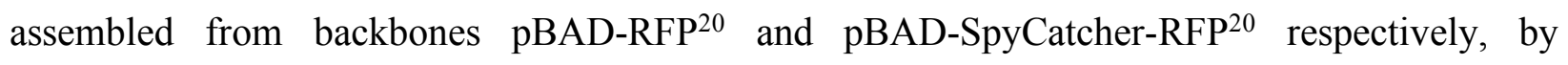
substituting the mRFP sequence with the GDH sequence. These plasmids were transformed into 
chemically competent BL21(DE3) cells (New England Biolabs) and single transformants selected for using ampicillin resistance.

GFP sequence:

ATGCGTAAAGGCGAAGAGCTGTTCACTGGTGTCGTCCCTATTCTGGTGGAACTGGAT GGTGATGTCAACGGTCATAAGTTTTCCGTGCGTGGCGAGGGTGAAGGTGACGCAAC TAATGGTAAACTGACGCTGAAGTTCATCTGTACTACTGGTAAACTGCCGGTTCCTTG GCCGACTCTGGTAACGACGCTGACTTATGGTGTTCAGTGCTTTGCTCGTTATCCGGA CCATATGAAGCAGCATGACTTCTTCAAGTCCGCCATGCCGGAAGGCTATGTGCAGGA ACGCACGATTTCCTTTAAGGATGACGGCACGTACAAAACGCGTGCGGAAGTGAAAT TTGAAGGCGATACCCTGGTAAACCGCATTGAGCTGAAAGGCATTGACTTTAAAGAA GACGGCAATATCCTGGGCCATAAGCTGGAATACAATTTTAACAGCCACAATGTTTAC ATCACCGCCGATAAACAAAAAAATGGCATTAAAGCGAATTTTAAAATTCGCCACAA CGTGGAGGATGGCAGCGTGCAGCTGGCTGATCACTACCAGCAAAACACTCCAATCG GTGATGGTCCTGTTCTGCTGCCAGACAATCACTATCTGAGCACGCAAAGCGTTCTGT CTAAAGATCCGAACGAGAAACGCGATCATATGGTTCTGCTGGAGTTCGTAACCGCA GCGGGCATCACGCATGGTATGGATGAACTGTACAAATAA

GDH sequence:

GACGTTCCGCTGACCCCGAGCCAGTTTGCGAAAGCGAAAAGCGAGAACTTCGACAA AAAAGTCATCCTGAGCAACCTGAATAAACCGCACGCTCTGCTGTGGGGTCCGGATA ATCAGATTTGGCTGACCGAACGCGCAACCGGTAAAATTCTGCGCGTTAACCCGGAA AGCGGCAGCGTTAAAACCGTCTTTCAGGTTCCGGAAATCGTTAACGACGCAGACGG 
TCAAAACGGTCTGCTGGGTTTTGCGTTTCATCCGGACTTCAAAAACAACCCGTACAT CTACATCAGCGGCACCTTCAAAAACCCGAAAAGTACCGACAAAGAGCTGCCGAATC AGACCATCATCCGTCGCTATACCTACAACAAAAGCACCGACACCCTGGAAAAACCG GTTGATCTGCTGGCAGGTCTGCCGAGTAGTAAAGATCATCAGAGCGGTCGTCTGGTA ATTGGTCCGGACCAGAAAATCTACTATACCATTGGCGATCAGGGCCGTAACCAACTG GCATACCTGTTTCTGCCGAACCAAGCACAACATACCCCGACCCAACAAGAACTGAA CGGCAAAGACTACCACACCTACATGGGCAAAGTTCTGCGTCTGAATCTGGACGGTA GCATTCCGAAAGACAACCCGAGCTTCAACGGCGTTGTTAGCCATATCTATACCCTGG GTCACCGTAATCCGCAAGGTCTGGCATTTACCCCGAACGGTAAACTGCTGCAGTCTG AACAGGGTCCGAATTCTGACGACGAAATCAACCTGATCGTTAAAGGCGGCAATTAC GGTTGGCCGAACGTTGCAGGCTATAAAGACGATAGCGGCTATGCATACGCGAATTA TAGCGCAGCGGCAAACAAAAGCATCAAAGACCTGGCCCAGAACGGTGTTAAAGTTG CAGCAGGCGTTCCGGTTACCAAAGAAAGCGAGTGGACCGGCAAAAACTTTGTTCCG CCGCTGAAAACCCTGTATACCGTCCAGGACACCTACAACTATAACGATCCGACCTGC GGCGAAATGACCTATATTTGCTGGCCGACCGTTGCACCGAGTTCTGCATACGTTTAC AAAGGCGGCAAAAAAGCGATCACCGGTTGGGAAAATACCCTGCTGGTTCCGAGTCT GAAACGCGGCGTTATCTTCCGCATCAAACTGGATCCGACCTATAGTACCACCTACGA CGATGCCGTTCCGATGTTCAAAAGCAACAACCGTTATCGCGACGTTATTGCAAGTCC GGACGGTAACGTTCTGTACGTTCTGACCGATACCGCAGGTAACGTTCAGAAAGACG ACGGTAGCGTTACCAATACCCTGGAAAATCCGGGTAGCCTGATCAAATTCACCTACA AAGCGAAATGA 


\section{Growth conditions for C. crescentus strains and BUD-ELMS}

Unless indicated otherwise, BUD-ELMs were grown by inoculating a single colony of $C$. crescentus strains into $80 \mathrm{~mL}$ of PYE in a $250 \mathrm{~mL}$ glass flask. These cultures were grown at $30^{\circ} \mathrm{C}$ at $250 \mathrm{rpm}$, and BUD-ELMs typically formed within $\sim 24-30 \mathrm{~h}$. To explore the effect of growth parameters on BUD-ELM size, the flask volume, shaking speed, and culture volume were varied from 125-250 mL, 0-250 rpm, and 30-80 mL, respectively. The complete list of conditions tested can be found in Table S3.

To test the ability of BUD-ELMs to re-seed their own growth, a piece of BUD-ELM grown under standard conditions was collected and transferred in a petri dish for 7, 14 or 21 days. The material dried out completely in less than $24 \mathrm{~h}$. A $\sim 0.3$ to $0.5 \mathrm{~cm}^{2}$ piece was broken off from the original material and inoculated into $80 \mathrm{~mL}$ of PYE. The culture was then incubated at $30^{\circ} \mathrm{C}$ at $250 \mathrm{rpm}$. We detected BUD-ELM formation in $48 \mathrm{~h}$ for material dried over 7 or $14 \mathrm{~d}$, and in $72 \mathrm{~h}$ for material dried over $21 \mathrm{~d}$.

\section{Expression and purification of SpyCatcher-GFP, and GFP from Escherichia coli}

Single colonies of E. coli BL21(DE3) harboring pSMCAF015 and pSMCAF016, for overexpression of GFP and SpyCatcher-GFP respectively, were inoculated in $25 \mathrm{~mL}$ of RM minimal media with $0.2 \% \mathrm{w} / \mathrm{v}$ glucose and $100 \mu \mathrm{g} / \mathrm{mL}$ ampicillin. After $\sim 16 \mathrm{~h}$ of growth at $37^{\circ} \mathrm{C}$ and $250 \mathrm{rpm}$, cells were used to inoculate $0.5 \mathrm{~L}$ of RM minimal media with $0.2 \% \mathrm{v} / \mathrm{v}$ glycerol, 100 $\mu \mathrm{g} / \mathrm{mL}$ ampicillin and $0.0004 \%$ antifoam (Antifoam 204) to a final $\mathrm{OD}_{600} \sim 0.05$. The cultures were allowed to grow at $37^{\circ} \mathrm{C}$ until mid-log phase. Protein production was induced with $0.2 \% \mathrm{w} / \mathrm{v} \mathrm{L-}$ arabinose with incubation at $30^{\circ} \mathrm{C}$ for $\sim 17 \mathrm{~h}$. 
Cells were harvested by centrifugation at $8000 \mathrm{xg}$ for $30 \mathrm{~min}$, resuspended in lysis buffer (50 $\mathrm{mM}$ Tris $\mathrm{pH} 8.0,300 \mathrm{mM} \mathrm{NaCl}, 5 \% \mathrm{v} / \mathrm{v}$ glycerol, and $10 \mathrm{mM}$ Imidazole) and lysed using using Avestin Emulsiflex C3 Homogenizer. The lysate was centrifuged at 12000xg for $1 \mathrm{~h}$ and the supernatant was collected for protein purification. The proteins were purified using Immobilized Metal Affinity Chromatography (IMAC) with a HisTrap FF column and buffers containing $50 \mathrm{mM}$ Tris $\mathrm{pH}$ 8.0, $300 \mathrm{mM} \mathrm{NaCl}, 5 \% \mathrm{v} / \mathrm{v}$ glycerol, and 10-250 mM Imidazole. After protein purity was confirmed by SDS-PAGE, the protein was dialysed into TEV-cleavage buffer (50 mM Tris $\mathrm{pH}$ 8.0, $0.5 \mathrm{mM}$ EDTA, $1 \mathrm{mM}$ DTT) and the $6 \mathrm{x}$ His-tag was cleaved using TEV protease by agitation at $4{ }^{\circ} \mathrm{C}$ for $4 \mathrm{~h}$. The cleaved protein was stored at $-80^{\circ} \mathrm{C}$ in $50 \mathrm{mM} \mathrm{NaPO}_{4} \mathrm{pH} 8.0,300 \mathrm{mM} \mathrm{NaCl}$ and $5 \% \mathrm{v} / \mathrm{v}$ glycerol.

\section{Imaging and Size Analysis of Macroscopic BUD-ELMS}

To image the BUD-ELMs, the bottom of a flask containing the BUD-ELM culture was imaged using a Canon EOS 77D camera. Flasks were positioned within a reflective photobox on a clear plastic surface such that the bottom of the flask was positioned approximately $11.5 \mathrm{~cm}$ above the camera lens. Flasks were illuminated from above by white LED strip lights, which were dampened by a thin white polyester fabric. For imaging of the air-water interface, each flask was instead imaged from above via a hole in the light box, and a black backdrop was positioned beneath the flask.

To determine the flat surface area of individual pieces of material under different growth conditions, images of flasks were separated into RGB channels using MATLAB. A subset of the blue channel images was then input into the image classification software ilastik, as a training set for the autocontext workflow. The first stage of training separated images into three different 
classifications: background, scattered material, and bundled material. Scattered material was defined as overlapping regions of small aggregates not associated with each other, whereas bundled material referred to larger, connected pieces of material. The second stage of training distinguished bundled material from the rest of the image. Both stages of training utilized all 37 features provided within the ilastik workflow. From the results of the training set, the second stage segmentation masks for all blue channel images were calculated, and loaded into MATLAB. From these masks, the flat area of each piece of material was calculated, and the top 5 percentile of size from each image was averaged to yield a representative size measurement. For each image, a conversion rate between pixels and millimeters was determined using the standard flask diameter as a reference point. Size measurements were averaged between samples and plotted with respect to their calculated $(\mathrm{d} 5)(\mathrm{kLa})(\mathrm{P} / \mathrm{VL})$ values.

\section{Imaging of Microscopic BUD-ELM structure using confocal microscopy}

Single colonies of BUD-ELM strain (RCC002) were inoculated in $30 \mathrm{~mL}$ PYE with 0.15\% $\mathrm{D}$-xylose - to induce the expression of mKate2, in a $125 \mathrm{~mL}$ flask and grown for $24 \mathrm{~h}$ at $30^{\circ} \mathrm{C}$ at a shaking speed of $250 \mathrm{rpm}$. BUD-ELMs of similar dimensions were collected and washed twice with $1 \mathrm{~mL}$ of $0.01 \mathrm{M}$ Phosphate-buffered saline (PBS), in a centrifuge tube. They were then incubated in $1 \mathrm{~mL}$ of $0.01 \mathrm{MPBS}$, at $30^{\circ} \mathrm{C}$, with the following staining agent: $80 \mu \mathrm{g}$ of SpyCatcherGFP or GFP for $1 \mathrm{~h}, 1 \%$ CongoRed (ThermoFisher Scientific-D275) or $100 \mu \mathrm{g}$ DiO(DiOC18(3) - 3,3'-Dioctadecyloxacarbocyanine Perchlorate) for $20 \mathrm{~min}$. Samples were washed 3 times with 1 $\mathrm{mL} 0.01 \mathrm{M}$ PBS and then a small amount was placed between a slab of PYE agarose $(1.5 \% \mathrm{w} / \mathrm{v})$ and a glass coverslip-bottomed 50-mm Petri dish with a glass diameter of $30 \mathrm{~mm}$ (MatTek Corporation). To acquire the low-magnification images (Fig. 2A - left panel), BUD-ELMs were 
embedded into 5\% w/v agarose and sliced. The slice was placed on a glass coverslip-bottomed 50mm Petri dish. For imaging, we used the Zeiss LSM800 Airyscan confocal microscope.

\section{In situ atomic force microscopy (AFM) imaging on living C. crescentus cells}

Poly-L-lysine coated silicon substrates were immersed in Falcon ${ }^{\mathrm{TM}}$ round-bottom polypropylene culturing tubes containing $3 \mathrm{~mL}$ fresh $C$. crescentus cell culture at an $\mathrm{OD}_{600}$ of 0.3 0.5. Culture tubes were then centrifuged at $3000 \times \mathrm{g}$ for $10 \mathrm{~min}$ to immobilize the cells onto the silicon substrate. The silicon substrate was washed with $2 \mathrm{~mL}$ of sterile PYE to remove looselybound cells before being mounted to a metal puck and transferred to the AFM sample stage. In situ AFM was performed on an Asylum Cypher AFM using soft tapping mode. A fluid cell and two syringe pumps were assembled to control liquid flow and PYE medium was supplied to maintain cell viability during imaging. The AFM probe consisted of a sharp silicon tip on a silicon nitride cantilever (BioLever mini, BL-AC40TS) with a spring constant of $0.09 \mathrm{~N} / \mathrm{m}$. Cells were imaged in native state without fixation. A 100-200 mV amplitude setpoint was used to apply minimum forces $(\sim 0.2 \mathrm{nN})$ to cells during imaging.

\section{AFM imaging on pellicle structures}

To bind BUD-ELM pellicle to a silicon substrate, a $2 \mathrm{~cm}^{2}$ precleaned silicon substrate was dipped into the pellicle forming cell culture with an entry angle of $\sim 60^{\circ}$ perpendicular to the water surface. The silicon substrate was then retrieved, and the pellicle structure was dried under $\mathrm{N}_{2}$ atmosphere for $2 \mathrm{~h}$. The dried pellicle structure on the substrate was mounted to a metal puck and transferred to the AFM sample stage. AFM imaging was performed on an Asylum Cypher AFM 
using soft tapping mode in air. A Tap-150 tip (BudgetSensors) with $5 \mathrm{~N} / \mathrm{m}$ force constant was used to image the pellicle structure.

\section{Immunoblot Analysis of BUD proteins}

Cultures of C. crescentus BUD-ELM strain (RCC002) were cultured in standard or stationary (not shaking) conditions. At $36 \mathrm{~h}$ for static cultures and at $24 \mathrm{~h}$ (sunken material phase) for shaking cultures, the supernatant of each culture was extracted and loaded onto a TGX Stain-Free ${ }^{\mathrm{TM}}$ gel (Bio-rad). After running, the gel was transferred to a $0.2 \mu \mathrm{m}$ nitrocellulose membrane and blocked for $1 \mathrm{~h}$ at room temperature with SuperBlock ${ }^{\mathrm{TM}}$ blocking buffer (Thermo Scientific). Membranes were then washed four times in TBST buffer before incubation in a 1:5000 dilution of Monoclonal ANTI-FLAG ${ }^{\circledR}$ antibody (Monoclonal ANTI-FLAG ${ }^{\circledR}$ M2-Peroxidase (HRP) antibody produced in mouse, clone M2, purified immunoglobulin, buffered aqueous glycerol solution) solution for 1 $\mathrm{h}$ at room temperature. Membranes were washed an additional four times in TBST buffer before Clarity Max Western ECL Substrate (Bio-rad) was applied and the membrane was imaged for chemiluminescence. Each sample lane represents an independent sample, grown from separate individual colonies.

\section{Building of Predictive Parameter for Material Size}

To describe the effect of shaking on BUD-ELM formation, a model was built based on the volumetric power input of a shake flask. The volumetric power input, defined as the rate of energy transfer into a shake flask per unit volume, was described by Büchs et al..$^{33}$ as:

$$
\frac{P}{V_{L}}=\frac{N e^{\prime} \cdot \rho \cdot n^{3} \cdot d^{4}}{V_{L}^{\frac{2}{3}}},
$$


Where $P$ is power, $V_{L}$ is culture volume, $n$ is shaking frequency, $d$ is the inner flask diameter,

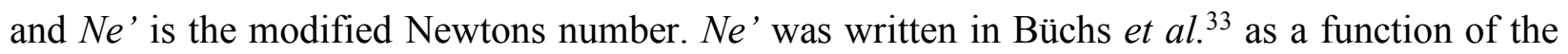
Reynolds number $R e^{\prime}$ in the following manner:

$$
\begin{gathered}
R e=\frac{\rho \cdot n \cdot d^{2}}{\eta_{a p p}}, \\
N e^{\prime}=70 R e^{-1}+25 R e^{-0.6}+1.5 R e^{-0.2},
\end{gathered}
$$

where $\eta_{\text {app }}$ is the apparent dynamic viscosity of the culture. To consider the impact of the airwater interface on BUD-ELM assembly, the volumetric power input was multiplied by the volumetric mass transfer coefficient of oxygen, defined dimensionally by Klöckner and Büchs ${ }^{34}$, as:

$$
k_{L} a=0.5 \cdot d^{\frac{73}{36}} \cdot n \cdot d_{0}^{\frac{1}{4}} \cdot V_{L}^{-\frac{8}{9}} \cdot D^{\frac{1}{2}} \cdot v^{-\frac{13}{54}} \cdot g^{-\frac{7}{54}}
$$

where $k_{L}$ is the transfer coefficient of oxygen, $a$ is the oxygen transfer surface area, $d_{0}$ is the shaking orbit diameter, $D$ is the diffusion coefficient, $v$ is the kinematic viscosity, and $g$ is the acceleration of gravity. To unify size measurements across different flask sizes, a correction factor of $d^{5}$ was applied. This new parameter was dubbed the "modified volumetric power", defined as:

$$
P_{v, a}=d^{5} \cdot k_{L} a \cdot\left(\frac{P}{V_{L}}\right)
$$

Calculations for $P_{v, a}$ assumed that the media parameters for viscosity $\eta_{\text {app }}$ and $\mathrm{v}$ and the diffusion coefficient $D$ were equal to that of water at standard growth temperature of $30^{\circ} \mathrm{C}$, which approximates culture conditions at inoculation.

\section{Parameter definitions and units}

$n$ - shaking frequency $\left(\mathrm{s}^{-1}\right) ; N e$ - modified Newton number (dimensionless); $P$ - power input (W); $R e$ - Reynold's number (dimensionless); $V_{L}-$ culture volume $\left(\mathrm{m}^{3}\right) ; \eta_{\mathrm{app}}-$ dynamic apparent 
viscosity (Pa s); $\rho$ - liquid density $\left(\mathrm{kg} / \mathrm{m}^{3}\right) ; d_{0}$ - orbital shaking diameter $(\mathrm{m}) ; D$ - diffusion coefficient $\left(\mathrm{m}^{2} / \mathrm{s}\right) ; v-$ kinematic viscosity $\left(\mathrm{m}^{2} / \mathrm{s}\right) ; g-$ acceleration of gravity $\left(\mathrm{m} / \mathrm{s}^{2}\right)$

\section{Rheological measurements}

The rheological properties of BUD-ELMs produced from strains RCC002, RCC004 and MFm152 (original BUD-ELM, $\Delta E L P_{60} \Delta S t p T a g$, and $\Delta r s a A_{1-250}$ strains, respectively) were evaluated on a strain-controlled rheometer (ARIES G2) equipped with an $0.1 \mathrm{rad} 8-\mathrm{mm}$ diameter cone plate. BUD-ELMs were grown in standard conditions. An approximate volume of 100-200 $\mu$ L of BUDELMs were collected into a $1.5 \mathrm{~mL}$ centrifuge tube and spun for $10 \mathrm{sec}$ at $3200 \mathrm{rcf}$ with a mini centrifuge (VWR $\left.{ }^{\circledR}, \mathrm{C} 0803\right)$. This allowed for the material to collect at the bottom as an homogeneous paste. The supernatant was removed and 150uL of fresh PYE were added on top of BUD-ELMs to prevent desiccation. Strain sweep experiments from 0.1 to $100 \%$ strain amplitudes were performed at a fixed frequency of $3.14 \mathrm{rad} / \mathrm{s}$. Frequency sweep experiments from 100 to $0.1 \mathrm{rad} / \mathrm{s}$ were performed at a $0.35 \%$ strain amplitude.

\section{Biosorption of $\mathrm{Cd}^{2+}$ to BUD-ELMS}

To measure the $\mathrm{Cd}^{2+}$ ability of BUD-ELMs, the $\Delta$ SpyTag strain was cultured in standard conditions. After growth for between 24 to 48 h, BUD-ELMs were harvested into sterile $2 \mathrm{~mL}$ tubes and lyophilized for $5 \mathrm{~h}$. Lyophilized BUD-ELMs were transferred to a metal-free $15 \mathrm{~mL}$ tube (VWR® Metal-Free Centrifuge Tubes, Polypropylene, Sterile) and incubated with $7 \mathrm{~mL}$ of 6 ppm $\mathrm{CdCl}_{2}$ (Sigma Aldrich 202908) in ddH2O for 90 min on an orbital shaker. After incubation, the $\mathrm{Cd}^{2+}$ concentration of the supernatant was measured by ICP-MS. Specifically, $5 \mu \mathrm{L}$ of the supernatant was diluted in $4.995 \mathrm{~mL} \mathrm{1 \%} \mathrm{HNO}_{3}$ with $5 \mu \mathrm{g} / \mathrm{mL}$ Indium (In), as standard for data 
analysis (Perkin Elmer N9303741). This diluted solution was run on a Perkin Elmer Nexion 300 ICP-MS with two isotopic measurements $\left(\mathrm{Cd}^{2+} 111, \mathrm{Cd}^{2+} 112\right)$ and $\mathrm{In} 115$ as the internal standard. Data was analyzed using Syngistix software. Samples were run in biological triplicates and data provided is mean with standard error.

\section{Functionalization of BUD-ELMs with PQQ-Glucose Dehydrogenase (GDH)}

Single colonies of E. coli BL21(DE3) harboring pSMCAF032 and pSMCAF029, for overexpression of GDH and SpyCatcher-GDH respectively, were inoculated in $25 \mathrm{~mL}$ of Terrific broth (TB) with $100 \mu \mathrm{g} / \mathrm{mL}$ ampicillin. After $\sim 16 \mathrm{~h}$ of growth at $37^{\circ} \mathrm{C}$ and $250 \mathrm{rpm}$, cells were used to inoculate $0.5 \mathrm{~L}$ of TB with $0.02 \%$ antifoam (Antifoam 204) and $100 \mu \mathrm{g} / \mathrm{mL}$ ampicillin to a final $\mathrm{OD}_{600} \sim 0.05$. The cultures were allowed to grow at $37^{\circ} \mathrm{C}$ until mid-log phase. Protein production was induced with $0.2 \% \mathrm{w} / \mathrm{v}$ L-arabinose with incubation at $30^{\circ} \mathrm{C}$ for $\sim 17 \mathrm{~h}$.

Cells were harvested by centrifugation at $8000 \mathrm{xg}$ for $30 \mathrm{~min}$, resuspended in lysis buffer $(0.02$ M PBS, $1 \mathrm{mM} \mathrm{MgCl}_{2}$ ) and lysed using Avestin Emulsiflex C3 Homogenizer. Lysates were centrifuged at $12000 \mathrm{xg}$ for $1 \mathrm{~h}$ and the supernatant was collected for BUD-ELM functionalization. The GDH (or SpyCatcher-GDH) in the lysate was reconstituted by adding a final concentration of $3 \mathrm{mM} \mathrm{Ca}^{2+}$ and $0.02 \mathrm{mM}$ PQQ and left in incubation for $15 \mathrm{~min}$ at $4^{\circ} \mathrm{C}$. BUD-ELMs, washed once with 0.01 M PBS, were incubated with reconstituted and non-reconstituted cell lysates for $3.5 \mathrm{~h}$ at $4^{\circ} \mathrm{C}$ and then washed three times with 0.01 M PBS. A small piece of functionalized BUD-ELMs (Fig. S11) was used for the colorimetric assay to detect GDH activity. 


\section{Colorimetric test to detect Glucose Dehydrogenase (GDH) activity}

The activity of GDH functionalized material was quantified with modified colorimetric 2,6dichlorophenol (DCPIP) assay ${ }^{35}$. A reagent solution of $48 \mathrm{~mL}$ MOPS buffer (10 mM, pH 7.0, 47 $\mathrm{mL}$ ), $1 \mathrm{~mL}$ DCPIP (20 mg dissolved in $5 \mathrm{~mL}$ of DI water), and $1 \mathrm{~mL}$ phenazine methosulfate (PMS) (45 mg dissolved in $5 \mathrm{~mL}$ of DI water) were prepared. Analytical samples were mixed with the reagent to $190 \mu \mathrm{L}$. The reaction was initialized by adding $10 \mu \mathrm{L}$ glucose (1M). Glucose consumption was correlated to the consumption of DCPIP (2:1 ratio), which was quantified colorimetrically by absorption at $600 \mathrm{~nm}$. A representative curve is shown in Fig. S12. The molar absorption coefficient of DCPIP at $600 \mathrm{~nm}$ was determined at $21 \mathrm{mM}-1 \mathrm{~cm}-1$. All the activity assays were performed in a $10 \mathrm{mM}$ MOPS buffer ( $\mathrm{pH} 7.0)$, including $50 \mathrm{mM}$ glucose, $558 \mu \mathrm{M}$ PMS, and $262 \mu \mathrm{M}$ DCPIP at room temperature. 
bioRxiv preprint doi: https://doi.org/10.1101/2021.11.12.468079; this version posted February 14, 2022. The copyright holder for this preprint (which was not certified by peer review) is the author/funder. All rights reserved. No reuse allowed without permission.

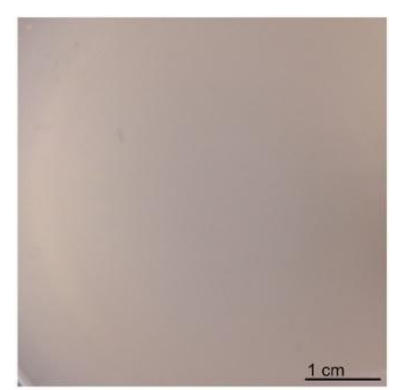

Fig. S1. The wild type strain does not form macroscopic aggregates under our growth conditions. Representative image of wild type strain (Mfm126) grown under standard conditions. 
A

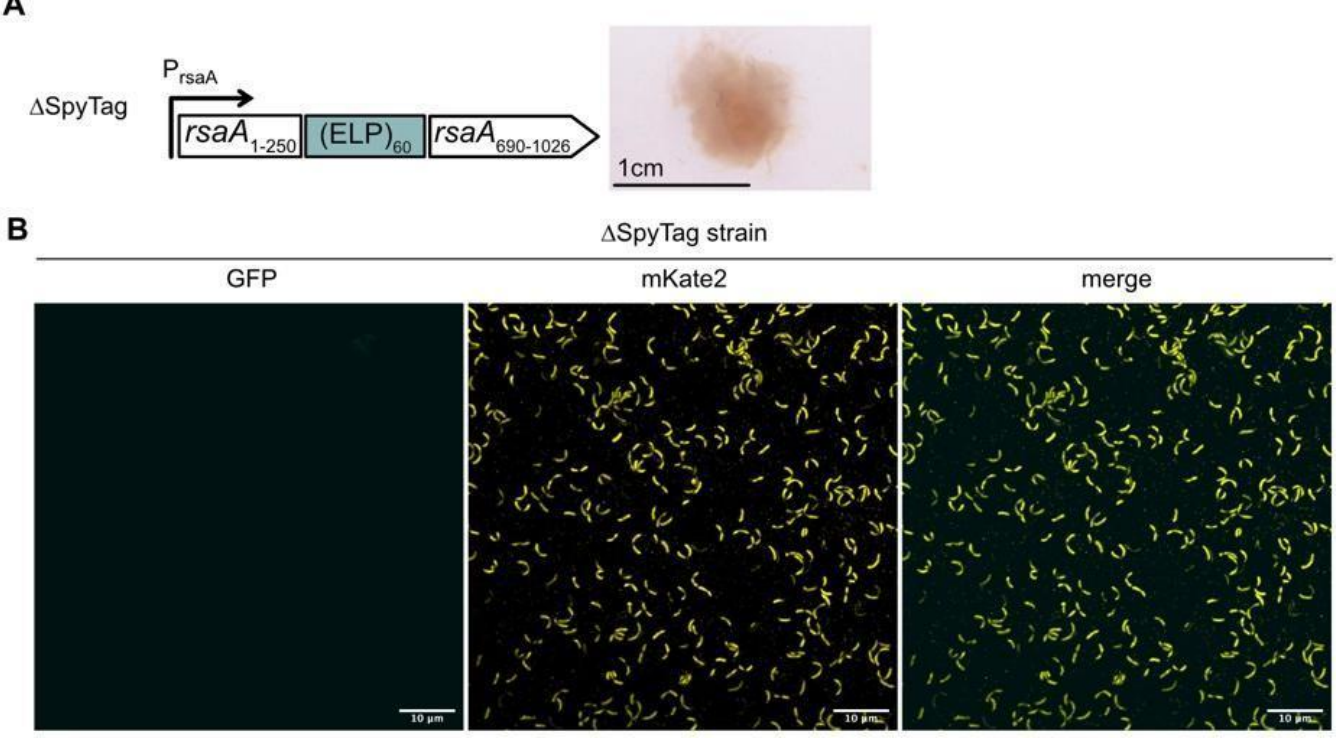

Fig. S2. The $\triangle$ SpyTag strain does not bind SpyCatcher-GFP. (A) Genetic constructs replacing the native $r s a A$ gene in the $\triangle$ SpyTag strain (left panel). Image of corresponding BUD-ELM (right panel) which is indistinguishable from the BUD-ELM containing the SpyTag. (B) Representative image of the $\Delta$ SpyTag single cells (yellow channel, mKate2) incubated with SpyCatcher-GFP (cyan channel, GFP), showing no significant SpyCatcher-GFP-cell binding. 

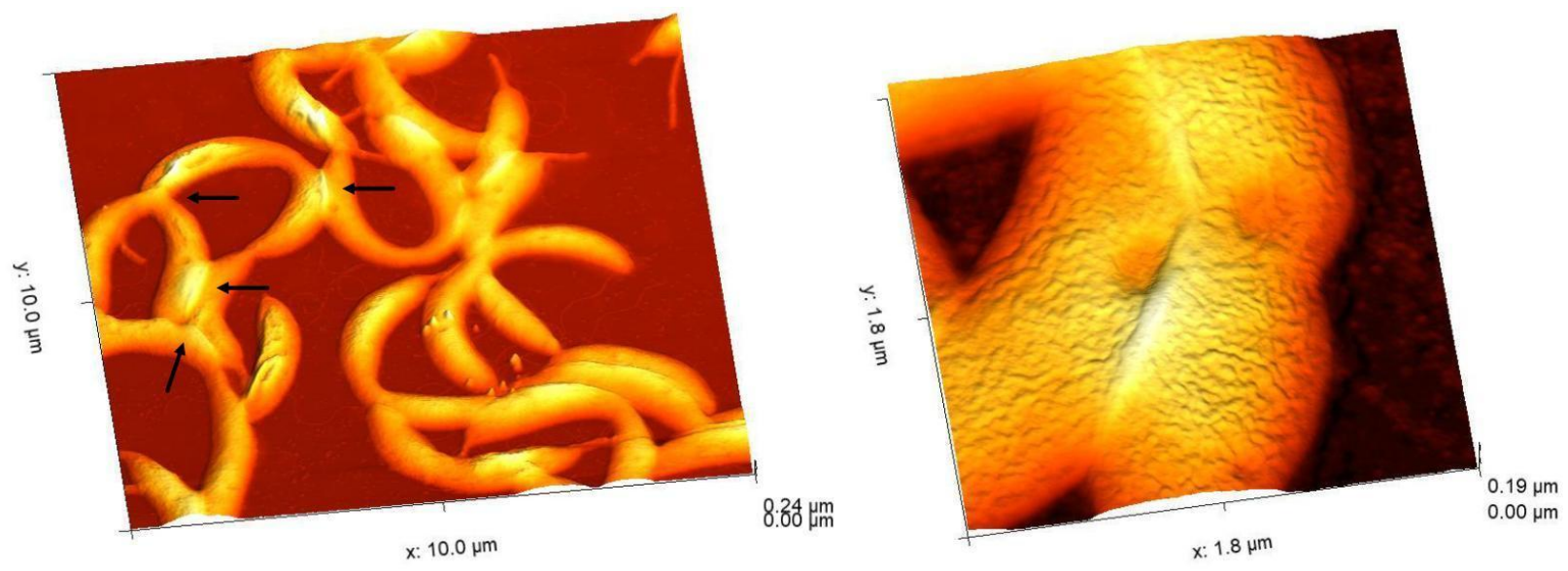

Fig. S3. AFM images show cell-cell interactions in the BUD-ELM strain. Representative AFM images at different magnifications of BUD-ELM strain single cells revealing the presence of cellcell interactions. The junctions of interacting cells show soft material accumulation. BUD-ELM cells also appear with the characteristic brush-like structure of the cell surface. 


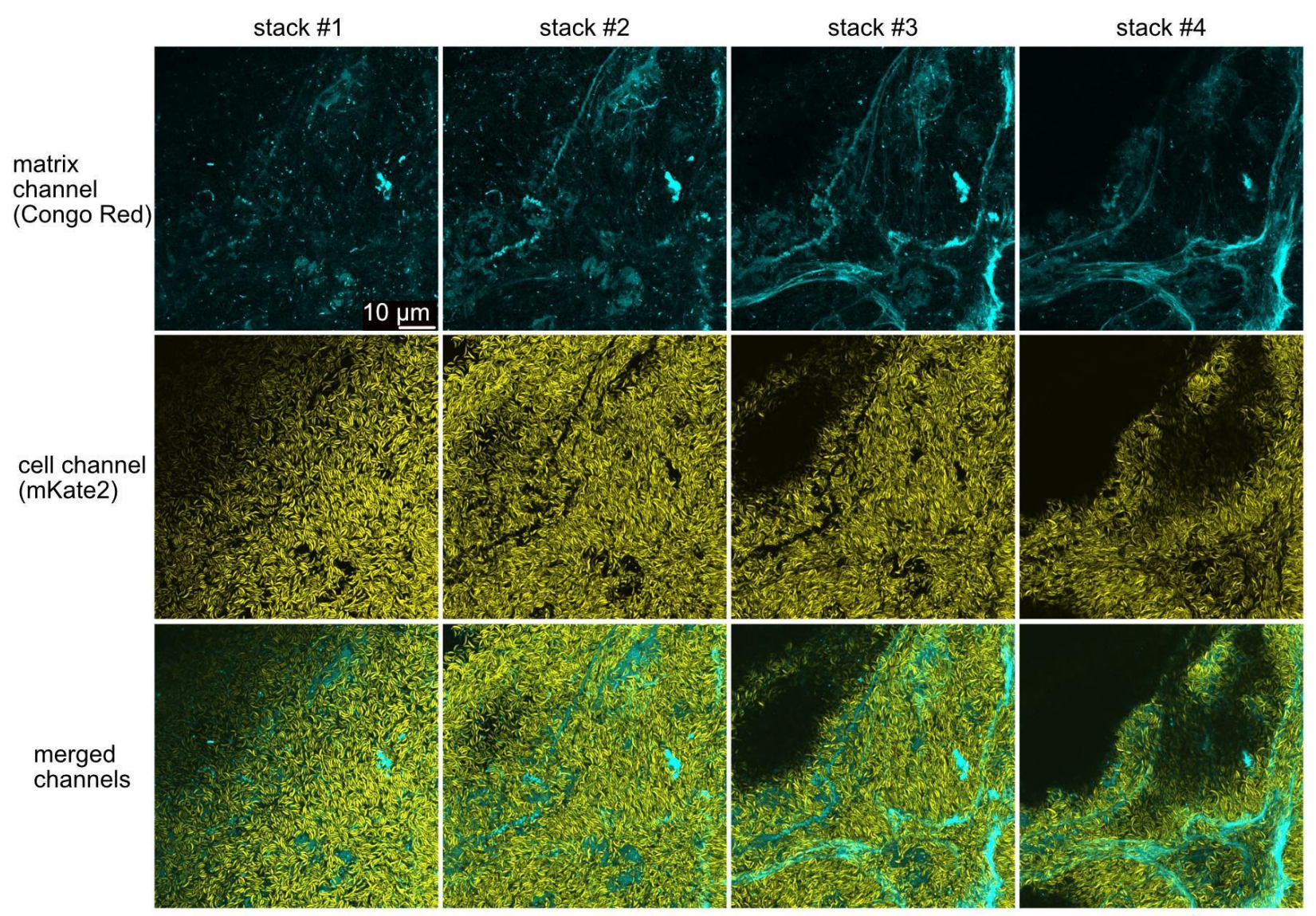

Fig. S4. The matrix is surrounded by cells within BUD-ELMs. Representative confocal microscopy stack images of the same BUD-ELM section. They show that the protein matrix, stained with Congo Red, is fully enclosed by cells. Scale bar applies to every image. 
A

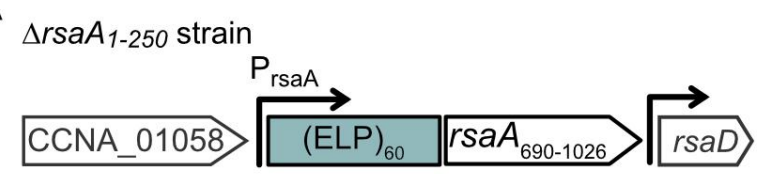

B

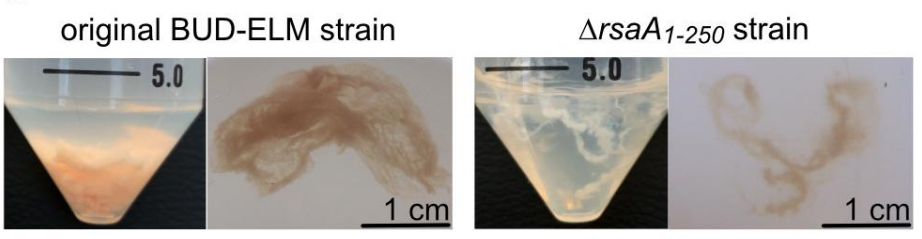

C
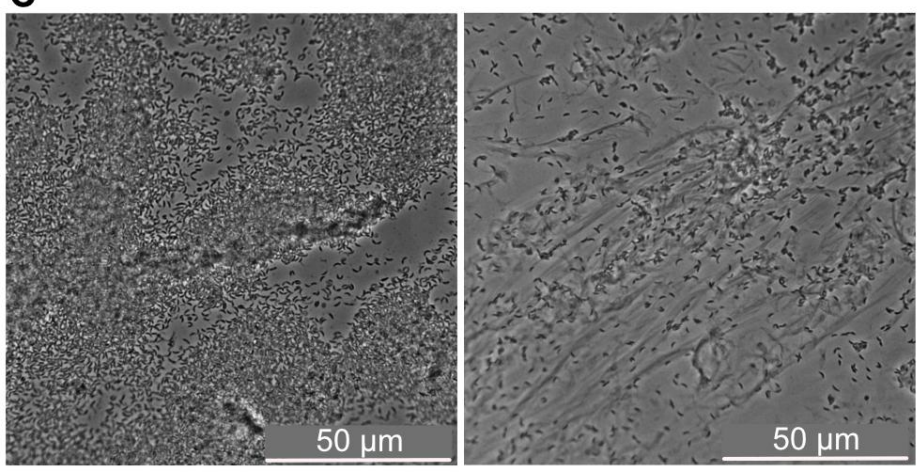

Fig. S5. $\Delta r s a A_{1-250}$ strain does not form a cell-rich BUD-ELM. (A) Synthetic construct replacing the native $r s a A$ gene in the $\Delta r s a A_{1-250}$ strain, showing the missing $\mathrm{N}$-terminal cell anchor domain of the BUD protein. (B) Representative images of BUD-ELMs (left panel) and material produced by the $\Delta r s a A_{1-250}$ strain (right panel) growing under standard conditions. The latter material has a distinct morphology (less cohesive) and color (lighter, white) compared to the original BUD-ELM. The material produced by the $\Delta r s a A_{1-250}$ strain appears also more buoyant. (C) Optical microscopy of the two materials show a clear difference in cell content, much higher in the original BUDELM. This is in agreement with the fact that $\Delta r s a A_{1-250}$ cells do not display the aggregating protein, and therefore cannot specifically adhere to the extracellular protein matrix. 


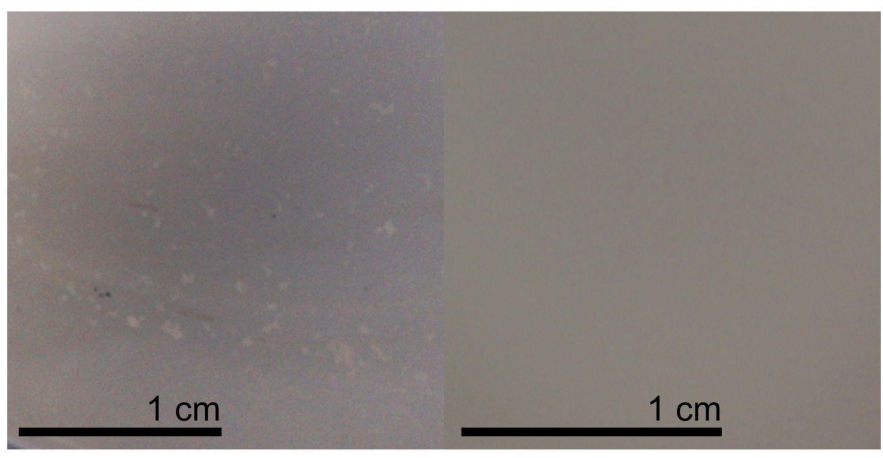

Fig. S6. Disruption of the liquid-air interface, through antifoam addition, prevents the formation of large BUD-ELMs. BUD-ELM strain grown under standard conditions with the addition of $0.04 \%$ antifoam showed small pieces of material at the bottom of the flask (left). No visible pellicle was observed at the air-water interface (right). 


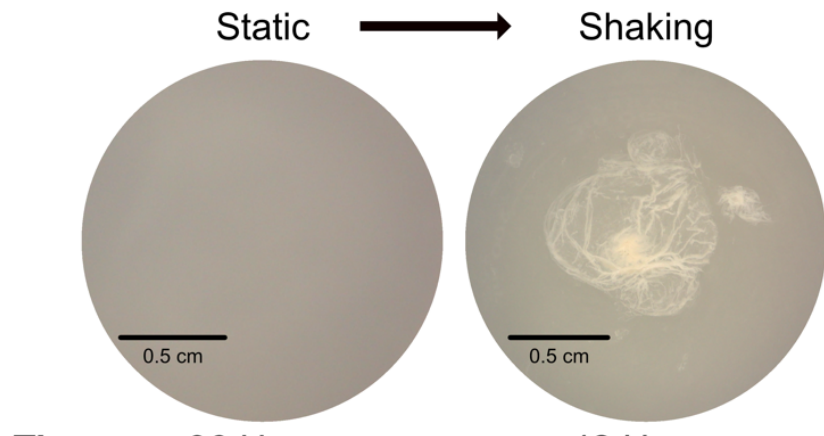

Time 36 Hours

42 Hours

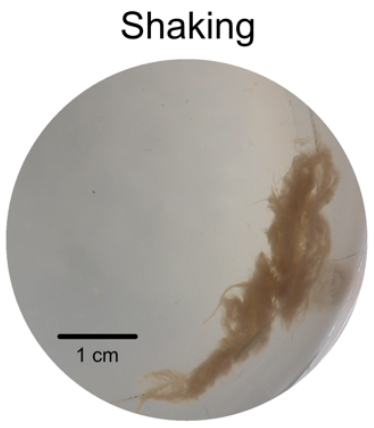

53 Hours

Fig. S7. Static-Grown Cultures form BUD-ELM in response to shaking. BUD-ELM strain grown in static conditions showed no material formation after $36 \mathrm{~h}$ (left image). When shaking was applied to cultures, pellicle (middle image) and final material (right image) formation were observed within 6 and $11 \mathrm{~h}$, respectively. 

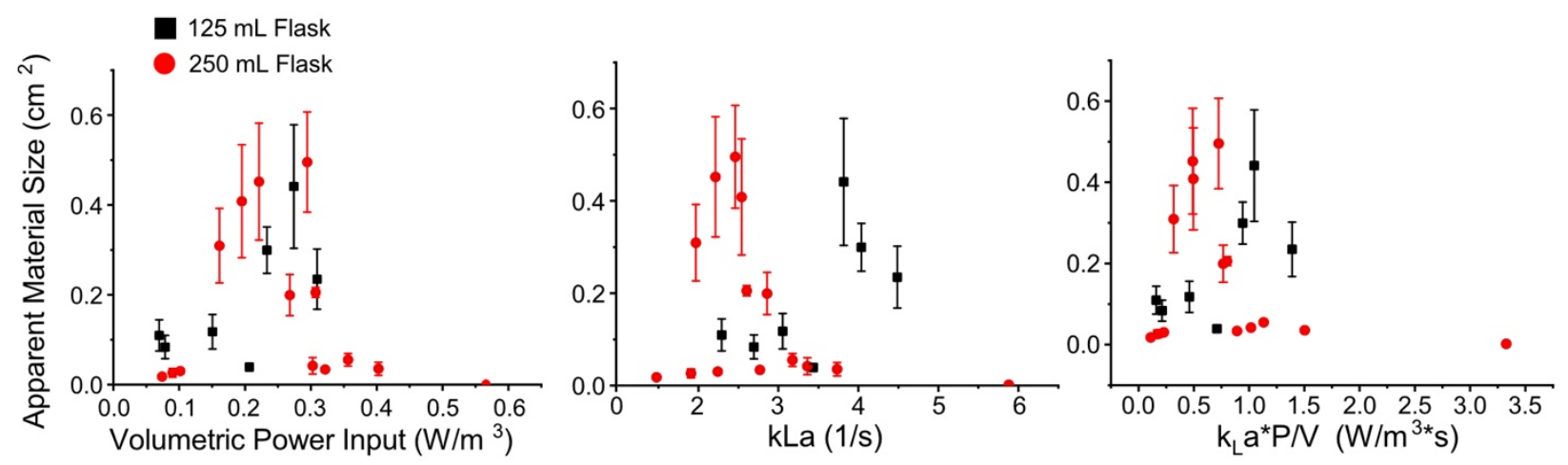

Fig. S8. Relationship between the major components of the Modified Volumetric Power and

Material Size. Flat surface area of BUD-ELMs was plotted against the Volumetric Power input (left), $\mathrm{k}_{\mathrm{La}}$ (middle), and the product of the two (right). None of these relationships provide a consistent metric for predicting large BUD-ELM size across flask sizes. Error bars represent standard error of at least three samples. X-axis label and legend apply to every graph. 


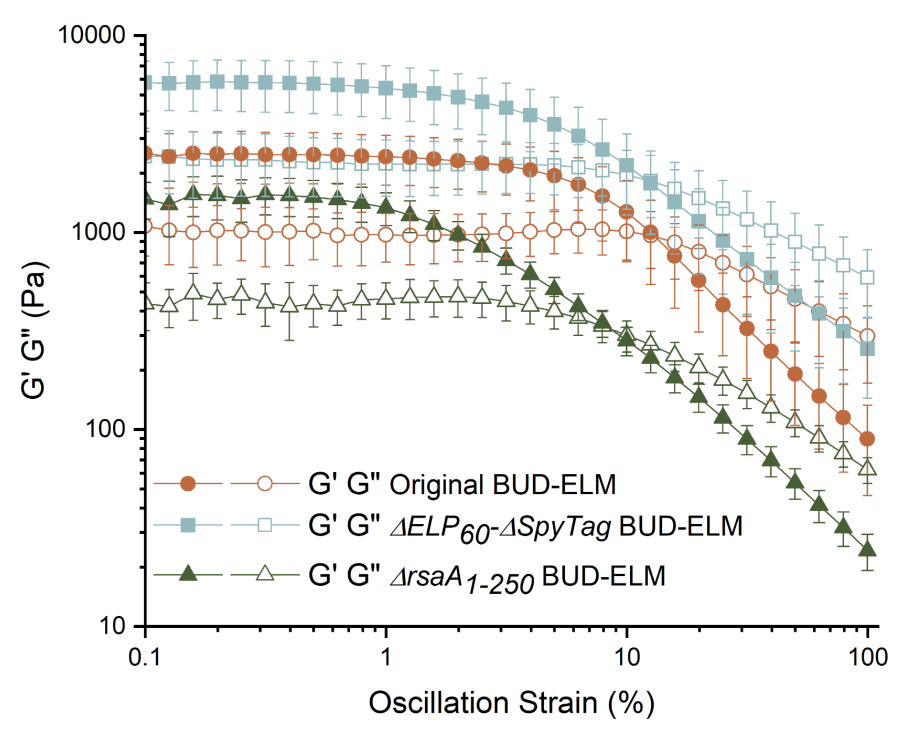

Fig. S9. Stress-strain curves of BUD-ELMs. Strain sweep measurements were acquired from $0.1 \%$ to $100 \%$ strain amplitude at a constant frequency of $3.14 \mathrm{rad} / \mathrm{s}$. Error bars represent $95 \%$ confidence intervals of at least five samples. From the amplitude sweep curves, we identified the linear viscoelastic region of the three BUD-ELMs and set the strain used to collect frequency sweep data (Fig. S10) to $0.35 \%$. 


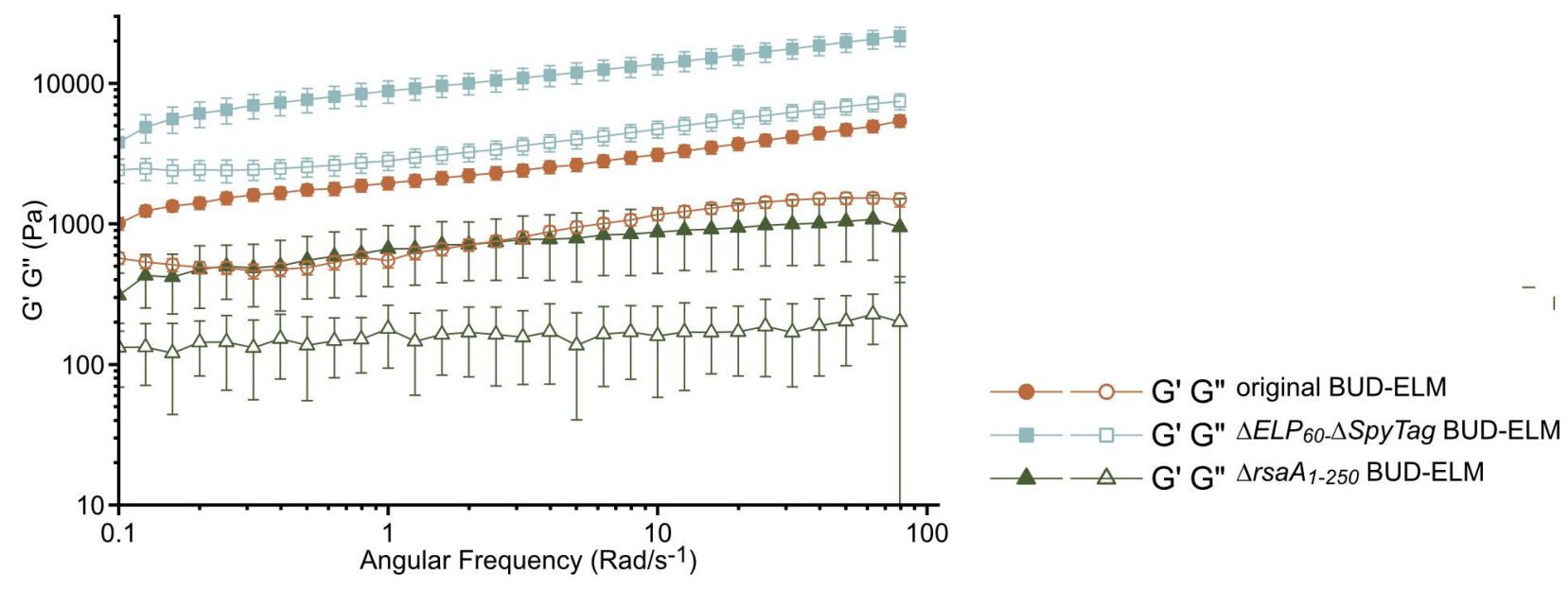

Fig. S10. Frequency sweep curve. Frequency sweep measurements were acquired from $0.1 \mathrm{rad} / \mathrm{s}$ to $100 \mathrm{rad} / \mathrm{s}$ at a constant strain amplitude of $0.35 \%$. Error bars represent $95 \%$ confidence intervals of at least five samples. 


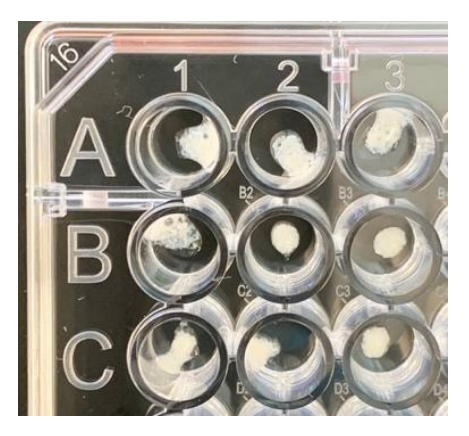

Fig. S11. Representative amount of BUD-ELM used the GDH activity colorimetric test. Each well contains a comparable amount of functionalized BUD-ELM. 

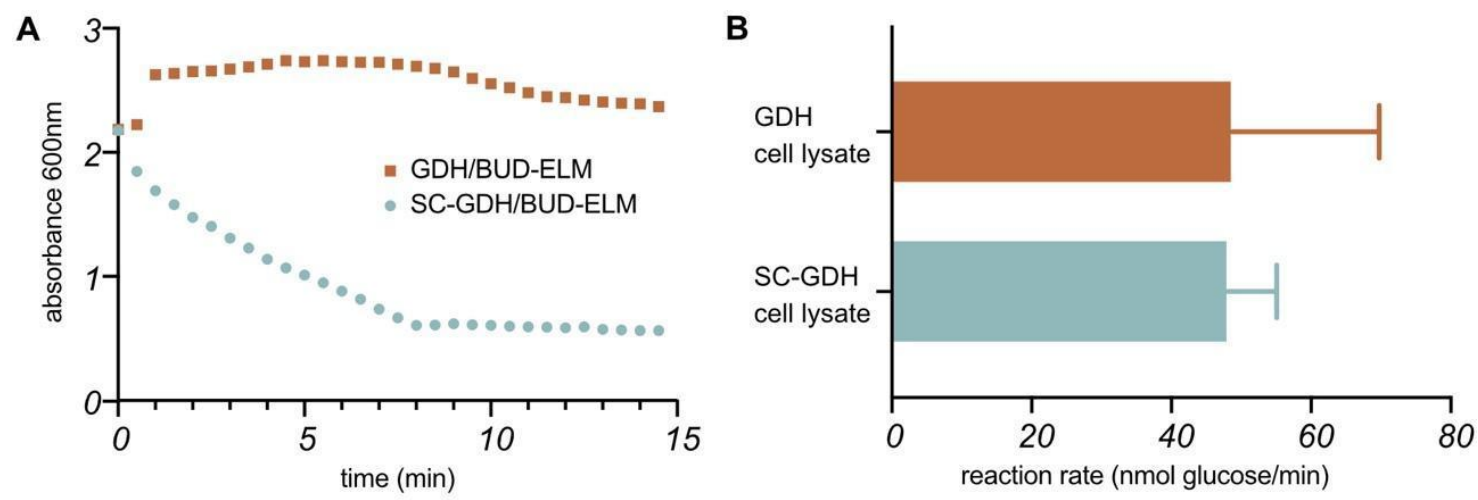

Fig. S12. Reaction rate of the cell lysate extracted from strains producing SpyCatcher-holoGDH and holo-GDH. (A) Representative kinetic curve of the enzymatic conversion of 2,6dichlorophenol-indophenol (DCPIP) by BUD-ELM incubated with SpyCatcher-holo-GDH (circles) or holo-GDH (squares). (B) Both strain lysate containing SpyCatcher-holo-GDH and holo-GDH show enzymatic activity. Error bars represent standard error of three samples. 


\begin{tabular}{|c|c|c|c|}
\hline Strain Name & $\begin{array}{l}\text { Strain } \\
\text { Designation }\end{array}$ & Source & genotype \\
\hline wild type & MFm126 & A. Persat et $a l^{35}$ & NA1000 $\triangle \operatorname{sap} A \because:$ Pxyl mKate2 \\
\hline$\Delta r s a A$ & MFm133 & M. Charrier et al ${ }^{20}$ & NA1000 $\triangle r s a A: \because$ Spec \\
\hline$\Delta r s a A_{1-250}$ & MFm152 & $\begin{array}{l}\text { M. T. Orozco- } \\
\text { Hidalgo et } a l^{21}\end{array}$ & $\begin{array}{l}\text { NA1000 } \triangle \text { sapA }: \because P x y l-m K a t e 2, \\
\Delta r s a A(1-689): \because E L P_{60}\end{array}$ \\
\hline $\begin{array}{l}\text { original } \\
\text { BUD-ELM }\end{array}$ & $\mathrm{RCC} 002$ & This work & $\begin{array}{l}\text { NA1000 } \Delta \text { sapA::Pxyl mKate2, } \\
\Delta r s a A(251-689):: E L \text { EL }_{60-S p y T a g,} \\
\text { (Fig. 1B). }\end{array}$ \\
\hline$\Delta E L P_{60} \Delta S p y T a g$ & $\mathrm{RCC004}$ & This work & $\begin{array}{l}\text { NA1000 } \Delta \text { sapA::Pxyl mKate2, } \\
\Delta r s a A(251-689),(\text { Fig. 1G). }\end{array}$ \\
\hline$\Delta$ SpyTag & $\mathrm{RCC005}$ & This work & $\begin{array}{l}\text { NA1000 } \Delta \text { sapA::Pxyl mKate2, } \\
\Delta r s a A(251-689): \because E L P 60,(\text { Fig. 1F). }\end{array}$ \\
\hline
\end{tabular}

Table S1. List of Strains used in this work. 


\begin{tabular}{|c|c|c|}
\hline Plasmid & Source & Description \\
\hline pSMCAF008 & This work & $\begin{array}{l}\text { Integration plasmid for strain RCC002. rsaA-N-terminus } \\
(800 \mathrm{bp}),(E L P)_{60}, \text { SpyTag, rsaA-C-terminus (800bp) }\end{array}$ \\
\hline pSMCAF017 & This work & $\begin{array}{l}\text { Integration plasmid for strain RCC004. rsaA-N-terminus } \\
\text { (800bp), rsaA-C-terminus (800bp) }\end{array}$ \\
\hline pSMCAF018 & This work & $\begin{array}{l}\text { Integration plasmid for strain RCC005. rsaA-N-terminus } \\
(800 \mathrm{bp}),(E L P)_{60}, r s a A-C \text {-terminus }(800 \mathrm{bp})\end{array}$ \\
\hline pSMCAF015 & This work & $\begin{array}{l}\text { pAra, HisTag-Tev-GFP; L-arabinose-induced expression of } \\
\text { GFP, used for protein purification (Fig. 1D). }\end{array}$ \\
\hline pSMCAF016 & This work & $\begin{array}{l}\text { pAra, HisTag-Tev-SpyCatcher-GFP; L-arabinose-induced } \\
\text { expression of SpyCatcher-GFP, used for protein purification } \\
\text { (Fig. 1D and Fig. 2A). }\end{array}$ \\
\hline pSMCAF029 & This work & $\begin{array}{l}\text { pAra, HisTag-Tev-SpyCatcher-GDH; L-arabinose-induced } \\
\text { expression of SpyCatcher-GDH, used for BUD-ELM } \\
\text { functionalization (Fig. 4F). }\end{array}$ \\
\hline pSMCAF032 & This work & $\begin{array}{l}\text { pAra, HisTag-Tev-GDH; L-arabinose-induced expression of } \\
\text { GDH, used for BUD-ELM functionalization (Fig. 4F). }\end{array}$ \\
\hline KR-12 & $\begin{array}{l}\text { Provided by } \\
\text { Kathleen R. Ryan }\end{array}$ & $\begin{array}{l}\text { pTac, GFP-mut3. IPTG-induced expression of GFP-mut3 in } \\
\text { C. crescentus. (Fig. } 2 \mathrm{~B} \text { - Congo Red staining). }\end{array}$ \\
\hline
\end{tabular}

Table S2. List of Plasmids used in this work. 


\begin{tabular}{|l|l|l|}
\hline Flask Volume (mL) & Shaking Speed (rpm) & Culture Volume (mL) \\
\hline 125 & 150 & 25 \\
\hline 125 & 150 & 30 \\
\hline 125 & 200 & 30 \\
\hline 125 & 225 & 25 \\
\hline 125 & 225 & 30 \\
\hline 125 & 250 & 25 \\
\hline 125 & 250 & 30 \\
\hline 250 & 150 & 50 \\
\hline 250 & 150 & 60 \\
\hline 250 & 150 & 80 \\
\hline 250 & 200 & 60 \\
\hline 250 & 200 & 80 \\
\hline 250 & 200 & 80 \\
\hline 250 & 225 & 50 \\
\hline 250 & 225 & 60 \\
\hline 250 & 225 & 80 \\
\hline 250 & 250 & 30 \\
\hline 250 & 250 & 50 \\
\hline 250 & 250 & 60 \\
\hline 250 & 250 & 70 \\
\hline 250 & 250 & 75 \\
\hline 250 & 250 & 80 \\
\hline & & \\
\hline
\end{tabular}

Table S3. List of Growth conditions (flask volume, shaking speed and culture volume) used to generate the model in Fig. 3D. 


\section{Methods References:}

33. Büchs, J., Maier, U., Milbradt, C. \& Zoels, B. Power consumption in shaking flasks on rotary shaking machines: II. Nondimensional description of specific power consumption and flow regimes in unbaffled flasks at elevated liquid viscosity. Biotechnology and Bioengineering $\mathbf{6 8}$, 594-601 (2000).

34. Klöckner, W. \& Büchs, J. Advances in shaking technologies. Trends in Biotechnology 30, $307-314$ (2012).

35. Olsthoorn, A. J. J. \& Duine, J. A. Production, Characterization, and Reconstitution of Recombinant Quinoprotein Glucose Dehydrogenase (Soluble Type; EC 1.1.99.17) Apoenzyme ofAcinetobacter calcoaceticus. Archives of Biochemistry and Biophysics 336, 42-48 (1996).

36. Persat, A., Stone, H. A. \& Gitai, Z. The curved shape of Caulobacter crescentus enhances surface colonization in flow. Nat Commun 5, 3824 (2014). 